\title{
Ueber die Isolirung der organischen Radicale;
}

\author{
von Dr. Edward Frankland.
}

Gelesen vor der Chemical Society of London.

\section{Ersle Abhandlung.}

Wenn man die Wichtigkeit in Betracht zieht, einen gewissen Beweis für die Existenz der bisher hypothelischen Radikale zu besilzen, welche einestheils einen Bestandheil jener basischen Verbindungen, der Aetherarten und Alkohole ausmachen, anderntheils mit Oxalsäure verbunden die mit der Formylsäure beginnende Reihe von Säuren bilden, so mufs es einigermalsen befremden, dals bis jetat so wenig Versuche angestellt worden sind, diese Körper zu isoliren, oder wenigstens die einfacheren Gruppen, in welche sie sich im Augenblick ihrer Abscheidung zerlegen, aufzufinden.

Durch die Methode, wonach es Bunsen *) gelang, das Kakodyl zu isoliren, war bereils ein Weg zur Abscheidung anderer Radicale vorgeschrieben; allein, einen erfolglosen Versuch von Löwig ausgenommen, durch die Einwirkung von Kalium auf Chloräthyl das Aethyl darzustellen, scheint die Sache nicht weiter beachtet worden zu seyn, bis es Kolbe **) gelang, auf einem ganz verschiedenen Wege, näılich durch die Elektrolyse der Valeriansäure, Valyl zu erballen.

Die Wirkung des Kaliums auf Cyanülhyl ***) liefs mich hoffen, dals durch die Anwendung eines weniger complicirten Körpers und eines weniger cleklropositiven Metalls das Radical isolirt werden könne, ohne zugleich in die Gruppen $\left(\mathrm{C}_{2} \mathrm{H}_{2}\right)$ und $\left(\mathrm{C}_{2} \mathrm{H}_{3}\right)$ zerspalten zu werden.

*) Diese Annal. Bd. XIII, S. 45.

**) Ebendaselbst Bd. LXIX, S. 257.

***) Ebendas. Br. LXV, S. 269. 
Die schwache Verwandischaft des Jods zu organischen Gruppen, seine starke Wirkung auf Metalle, und die verhältnifsmïlsig niedrige Temperatur, bei welcher sich seine organischen Verbindungen, unter Ausscheidung von freiem Jod, zersetzen, veranlalsten mich, die Verbindungen dieses Elements als besonders für derartige Versuche geeignet zu halten, und obgleich, aufser Kalium und Natrium, vielleicht kein Metall auf diese Körper wegen ihres niederen Kochpunktes einwirkt, so hielt ich es doch für wahrscheinlich, dafs, wenn das Metall und das Jodid unler höherem Druck eincr regelmälsig zunehmenden Temperalur ausgesetzt werden, man einen Punkt errcichen wïrde, wo die Verwandtschaft des Metalls zum Jod die des Radicals zu demselben Elemente überwiegt und die Zersetzung ler organischen Jodverbindung vor sich geht; übrigens war zu vermulhen, dafs die Zusammenselzung der Producte sehr von der Temperalur, vielleicht auch in cinem gewissen Grade von den Eigenschaften des angewandten Metalls abliängen würde.

Da mir dic Kenntnils des Aethyls vor allen anderen Radicalen das meiste Interesse darzubieten schien, so habe ich dessen Isolirung aus dem Jodähyl zunächst meine Aufmerksamkeit zugewandt. Zur Darstellung des letzleren diente folgendes Verfahren : In einem mit eiskaltem Wasser umgebenen Gefäls wurden 7 Theile Phosphor mit 35 Theilen absoluten Alkohol übergossen und der Mischung nach und nach in kleinen Quantitäten 23 Theile Jod zugesetzt. Die nach beendeter Zerselzung von dem gebliebenen festen Rückstande abgegossene Flüssigkeit wurde alsdann im Wasserbade destillirt, das Destillat wiederholt mit Wasser gewaschen, darauf mit so viel Jod versetzt, bis es schwach davon gefärbt blieb und über Chlorcalcium getrocknet. Um es vom Wasser, freiem Jod und Jodwasserstoffsäure vollkommen zu befreien, war noch einc dreimal wiederholte Rectification über jedesmal erneutem Gemenge von Chlorcalcium, Quecksilber und reinem Bleioxyd erforderlich. Der 
Siedepunkt des so gereinigten Jodäthyls betrug alsdann bei 746,5mm Druck, wenn das Thermometer unmiltelbar über der Oberflüche stand, $71,6^{\circ} \mathrm{C}$; war hingegen die Kugel des Thermometers in die Flüssigkeit getaucht, so blieb die Temperalur constant bei $72,2^{\circ}$ C. Sein specifisches Gewicht war 1,9464 bei $16^{\circ} \mathrm{C}$.

Mit Kupferoxyd verbrannt *) lieferten 0,5618 Grm. 0,3190 Grm. Kohlensäure und $0,1607 \mathrm{Grm}$. Wasser, woraus folgende procentische Zusammenselzung folgt :

\begin{tabular}{|c|c|c|c|c|c|}
\hline \multirow{2}{*}{\multicolumn{3}{|c|}{4 Aeq. Kohlenstoff }} & & berechnet & gefunden \\
\hline & & & 300,0 & 15,42 & 15,48 \\
\hline & $m$ & Wasserstoff & 62,5 & 3,20 & 3,18 \\
\hline & » & Jod & 1585,5 & 81,38 & 》 \\
\hline
\end{tabular}

Um das Jodälhyl in Berührung mit verschiedenen Metallen solchen Temperaturen auszusetzen, welche scinen Kochpunkt bedeulend übersteigen, und ur zugleich alle sich eniwickelnden Gase zu crhalten, wurde folgendes Verfahren beobachtet. Harte böhmische Glasröhren von ungefähr 1,3mun Dicke, 10mm Durchmesser und etwa 12 Zoll Länge wurden am einen Ende vor dem Löthrohr vorsichlig zugeschmolzen, so dafs das Glas möglichst gleiche Dicke behielt. Dic Metalle wurden darauf im granulirten, oder sonst fein zertheillen Zustande durch das offene Ende der Röhre hineingebracht und dicses dann ebenfalls zur Dicke cines Strohhalms ausgezogen. Es ist nothwendig, den hinteren Theil $(b)$ der ausgezogenen Röhre Fig.I auf's Neue bis zur Weite eines Haarröhrchens zu verengen. Diefs wird dadurch

*) Bei dieser Analyse wurde das vordere Ende der Verbrennungsröhro circa vier Zoll lang mit metallischem Kupfer angefüllt und während der ganzen Operation so genau wie möglicl gerade unter einer bei Tageslicht sichtbaren Glühhitze crhalten. Nach der Verbrennung liefs sich in der im Kaliapparate befindlichen Flüssigkeił keine Spur von Jod nachweisen. 
Fir. I. erreicht, dafs man diesen Theil clwa einen Zoll lang in die heilseste Stelle der Flamme

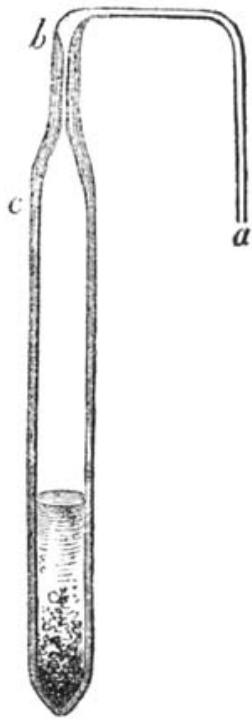
der Glasbläserlampe bringt und darin das Glas zusammenfallen läfst. Der vordere Theil der ausgezogenen Röhre ist, wie Fig. I zeigt $*$, dicht hinter der verengten Slelle zweimal rechtwinklich gebogen. Wird darauf das Ganze erwärmt, und das offene Ende der Röhre (a) in das Jodälhyl gelaucht, bis durch dic erfolgte Abkühlung cinc abgenessene Menge, elwa 6 Gramme, cingesogen ist, so bedarf es nur noch einer Verbindung desselben durch eine Kaulschukröbre mit der Luflpumpe, und so lange fortgesetzler Evacuirung, bis das cingeschlossene Jodällyl zu sieden beginnt, um die Röhre nach dem Abschmelzen bei (b) vollkommen luftrei zu erhallen. Sie wird darauf in cin Oelbad gelegt und erhitzt.

\section{Einwoirkung des Zinks auf Jodäthyl.}

Ein vorläufiger Versuch auf die eben beschrielsene Weise angestellt, zeigte, dals die Zerselzung des Jodäthyls durch Zink bei einer Temperatur von circa $150^{\circ} \mathrm{C}$. ihren Anfang nimmt und mit ziemlicher Geschwindigkeit fortschreitet, wenn das Mctall eine grofse Oberfläche darbietet; wcilse Krystalle scłzen sich allmälig an das Glas und das Zink an, während eine farblose, leicht bewegliche Flïssigkeit zurïckbleibt, im Volum etwa nur der Hälfte des angewandten Jodälhyls gleich, und dem Ansehen nach sehr davon verschieden. Das Aufhören des Kochens bald nach dem Anfange der Zerselzung zeigt an, dafs sich ein Gas

*) Bei meinen Versuchen ragten die Metallstücke etwa 2 Zoll über die Flüssigkeit heraus. 
oder ein Dampf von hoher Tension entwickelt hat. $\Lambda l s$ der Apparat elwa zwei Stunden lang jener Temperalur ausgeseizt gewesen war, schien die Zerselzung beendet zu seyn. Die Röbre wurde sofort aus dem Oelbade entfernt und crkalten gelassen; beim Abbrechen der haarengen Spitze unter Wasser entströmte dem Rohre clwa sein 40faches Volumen Gas, während die ganze Menge der oben erwälnten leicht beweglichen Flüssigkeit verschwand. Das Gas halle cinen starken älherartigen Geruch, brannte mit heller Flamme und wurde von frisch ausgekochtem absoluten Alkohol schnell und vollkommen absorbirt. Die in dem Rolr zurückbleibende weifse Krystallmasse löste sich in destillirlem Wasser unter starkem Aufbrausen auf, bewirkt durch die Entwickelung eines Gases, welches in seinen Eigenscluaften dem obigen selır nahe stelt. Die so erhaltene Lösung der Krystalle hatte alle Eigenschaften einer Lösung von Jodzink, und schien, aufser einer Spur unzersetzten Jodäthyls, keine organische Subslanz zu cnthalten.

Fig. Il.

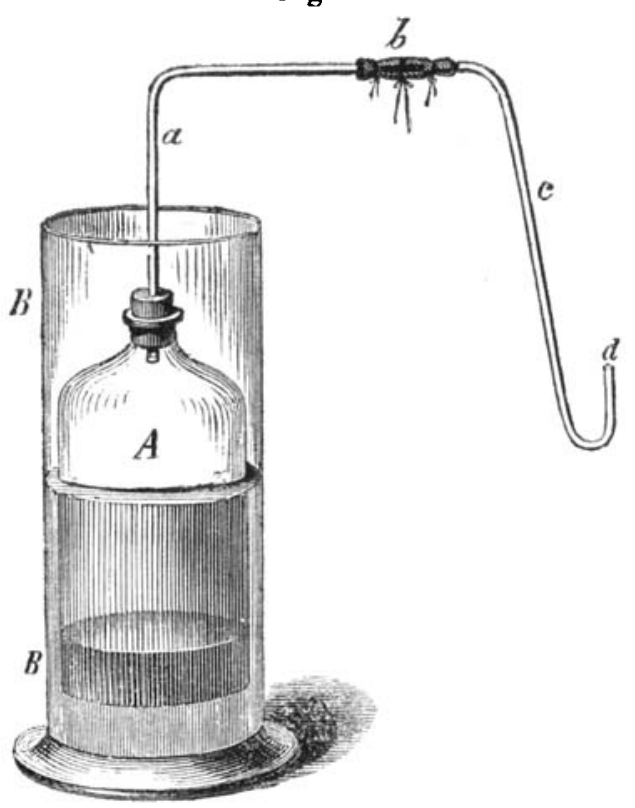

Um das Gas aufzufangen, bedienle ich mich des in Fig. II abgebildeten Apparals. $\boldsymbol{A}$ ist eine unten offene Glasglocke, welche sich leicht in den Cylinder $B B$ schieben lälst und deren Hals durch einen guten Kork verschlossen ist, durch den dieGlasröhre $(a)$ in jene mündet. Letzlere ist oberhalb des 
Korks in rechten Winkel gebogen und durch das Kaulschukröhrchen (b) mit der Gasentwickelungsröhre (c) verbunden. In der Kautschukverbindung (b) und zwischen den Röhren (a) und (c) befindet sich ein Glasstäbchen, einen halben Zoll lang und von etwas geringerem Durchmesser, als die biegsame Röhre, so dafs durch eine aufsen angebrachte Schnur der Raum zwischen dem Glasstäbchen und dem Kautschuk ohne Nachtheil für lelzteres nach Belieben geöffnet oder geschlossen werden kann.

Es ist durchaus nothwendig, das Gas über Wasser aufzufangen und es wenigstens 24 Stunden lang stehen zu lassen, damit der Dampf des unzersetzlen Jodälhyls vollständig absorbirt wird. Da während dieser Zeit eine Diffusion zwischen dem Gase und der almosphärischen Luft nicht zu vermeiden scyn würde, und sogar auch durch eine Oclschicht nicht beseiligt werden kann, das Eindringen jeder Spur von Saucrstoff aber um so mehr vermieden werden mufs, als ich fand, dafs die $\mathbf{A b}$ sorption desselben durch Phosphor in diesem Gasgemenge nur bei einer 'Temperatur von Statten geht, dic bedeutend hüher ist, als der Schmelzpunkt des Phosphors, so bediente ich mich anslatt des reinen Wassers einer sehr verdiinnten Lösung von Schwefelkalium. Es wurde hiermil ein geräumiges Porcellangefäls soweit angefüllt, dafs der Cylinder $B B$ bequem dariı untergelaucht werden konnte. Durch Ocffnen des Ventils (b), Eintauchen der Glocke $\boldsymbol{A}$ in jene Flüssigkeil und Saugen mit dem Munde bei $(d)$ wurde auch dieser Apparat ganz damit gefüllt und alle Luft entfernt.

Nachdem das Ventil (b) wiederun geschlossen war, wurde eine der vorher beschrielıenen, in Oelbade crhitzten Zersetzungsröhren, worin elwa 6 Gramme Jodäthyl der Einwirkung des Zinks unterlegen hatten, unter der Glocke an dem haarfeinen Theile mit einer Feile geöffnet. Das stark conprimirle Gas entwich dabei anfangs mit grofser Heftigkeit, hernach immer langsamer, so dafs die Ausströmung beinahe eine Viertelslunde 
andaucrte. Die so mit Gas gelüllte Glocke wurde alsdann unter jener alkalischen Flüssigkeit in den Cylinder $B \boldsymbol{B}$ gebracht und mit diesem aus dem Porcellangefäfs herausgehoben. Das auf diese Weise durch eine schmale Flïssigkeitsschicht abgesperrte Gas, welches ich durch a bezcichnen will, wurde dann 24 Stunden sich überlassen, ehe es zu weiteren Versuchen diente.

Nachdem die Röhre das darin comprimirte Gas ausgegeben halle, wurde sie zwölf Stunden lang stehen gelassen und darauf bei $(c)$, Fig. I, wo sie ihre ursprüngliche Weite noch besals, abgeschnilten, der darin enthaltene Salzrückstand mit Wasser übergossen und dic Oeflnung rasch durch einen mit ciner Gasleilungsröhre verschenen Kork verschlossen. Erst nachdem durch die beim Eingiefsen von Wasser eintretende schr lebhafte Gasentwickelung alle in die Rühre zuvor eingedrungene Luft ausgetrieben war, wurde jenes Gas, welches ich durch $\beta$ bezeichnen will, in einem dem obigen ähnlichen Apparale und unter Beobachtung dersclben Vorsichtsmafsregeln aufgefangen.

Um dic Natur des ersten Gases $\alpha$ zu crmitteln, versuchte ich zunächst sein specifisches Gewicht zu bestimmen. Hierzu diente cin leichter, durch einen eingeschliffenen Glasslöpsel verschliefsbarer Kolben von circa $200 \mathrm{Cbc}$. Inhalt, dessen Hals auswendig mil ciner genauen Millimeterscale versehen war. Auf dem Boden des Kolbens befanden sich, um das einzulassende feuchte Gas zu trocknen, cinige Stücke geschmolzenen Kali's, welche durch Befeuchtung mit ein wenig Wasser und Erwärmen an der Innenwand des Gefäfses befestigt waren. Der Ballon wurde darauf mil Quecksilber gefüllt und verkehrt in dic Quecksilberwanne gestellt. Durch Nicderdrüicken der Glocke $\boldsymbol{A}$ in dem umschliefsenden Cylinder $B B$ und Oeffnen des Kautschukventils (b) wurde zunächst das Wasser aus der unter Quecksilber mündenden Gasleitungsröhre (c) ausgetricben und das Ende der lelzteren erst dann in die Miindung des Kolbens cingefiihrt, nachdem einige Gasblasen frei in dic Luft ausgelrelen waren und die 
Oberfläclse des Quecksilbers vom aufschwimmenden Wasser sorgfältig gereinigt war. Um der unbequemen Nolbwendigkeit zu enlgehen, in $B B$ cinen starken Druck anzuwenden, ist das äufserste gebogene Ende $(d)$ der Gasleitungsrölre so beschaffen, dafs die Mündung derselben innerhalb des Halses vom Ballon elwas höher steht, als die Oberflüche des äufseren Quecksilbers reicht. Es bedarf dann in $B B$ nur einer wenige Zoll holen Wassersäule, un die Capillarität der engen Röhre zu überwinden.

Nachdem so viel Gas in den Glasballon eingetreten war, dafs die innere Quceksilberfliche mit der äufseren in Niveau stand, wurde dic wcitere Zuleitung unterbrochen, dic Gasleitungsröhrc aus dem Halse des Kolbens entfernt und der Rest des Gases in cinen anderen Behälter über Quecksilber übergefüllt, um es noch zu cudionctrischen Versuchen zu benulzen. Die verschicdenen Ablesungen wurden erst nach mehreren Stunden vorgenommen, wenn man annehmen konnte, dafs das im Ballon cingeschlossene Gas die Temperatur der ungebenden Luft besafs und durch dic Kalistiicke vollständig entwässert war. Es wurden folgende Zahlen crhalien :

Temperatur des Zimmers . . . . 6,20 C.

Barometerstand . . . . . . .760,9mm.

Höhe der inneren Quecksilbersäule . . 19,4 $\mathrm{mm}$.

Gewicht des mil Gas gefiillten Ballons 54,6213 Gru.

Temperalur in der Waage . . . $7,8^{\circ} \mathrm{C}$.

Gewicht des mit Luft gefüllten Ballons 54,4838 Grur.

Temperatur in der Waage . . . $8,0^{\circ} \mathrm{C}$.

Inhalt des Gefälses . . . . . . 211,23 Cbc.

Hieraus ergiebt sich das specifische Gewicht des Gases zu 1,5250 .

Zur ferneren Ermiltelung der Zusammenselzung des Gases bedienle ich mich der von Bunsen eingeführten eudiometrischen Methoden, wornach die Bestimmungen mil einer bisher 
unerreichten Genauigkeit ausgeführt werden können. Ich ergreife hicr zugleich freudig die Gelegenheil, dem Herrn Professor B unsen, welcher mir während der ganzen Dauer dieser Untersuchung sein Laboratorium, so wie besonders scine ausgezcichneten Apparate zur Untersuchung von Gasen bereitwilligst zur Verfügung stellte, dafür meinen wärınsten Dank öffentlich auszusprechen.

Von dem zur eudiometrischen Untersuchung beslimmten Gase wurde ein Theil in cin kurzes Eudiometer übcrgefüllt, darin durch eine Kalikugel gelrocknet und darauf scin Volum mit Beachtung aller nöthigen Vorsichtsmafsregeln abgelesen. Sodann wurde eine frisch ausgeglühte und mit slark rauchender Schwefelsäure getränkte Coakkugel eingeführt und eine Zcit lang damit in Berührung gelassen. Zur Absorption der dabei sich enlwickelnden schwefligsauren Däınfe und zunn völligen Trocknen dienten nach einander eingebrachle Kugeln von feuchtcm Mangansuperoxyd und gesehmolzenem Kalihydrat. Nachdem das Volumen des rückstündigen Gases auf's Neue abgelesen und ein Theil desselben zur Verbrennung mit Sauerstoff in cin gröfseres Eudiometer übergefüllt war, wurde der abermals genau gemessene Rest über Quecksilber mit cinem ungefähr gleichen Volumen frisch ausgekochten, noch warmen absoluten Alkohols zusammengebracht, damit geschïllelt und dann einige Stunden stehen gelassen. Die dabci unabsorbirt gebliebene kleine Menge des Gases wurde mit Beriicksichligung der Correction für dic Spannung des Alkoholdampfs als reincr Stickstoff in Rechnung gebracht. Der in das gröfsere Eudiomeler zuvor übergefuillte Theil des mil Schwefelsäure bchandelten Gases wurde in diesem mit gemessenen Quantitäten Sauerstoff und almosphärischer Luft ${ }^{*}$ ) durch den elcktrischen Funken verbrannt, dic erzeugte

*) Die Analyse diescs Gases bot einjge Schwierigkeiten dar, da seine auf gewöhnliche Weise vorgenommene Verbrennung mit Sauerstolf 
Kohlensüure durch eine Kalikugcl ausorbirt und der Rest mit einer bekannten Mlenge trocknen Wassersloffs im Uebermaals verbrannt, um zı prüfen, ob die ersterc Verbrennung vollständig gewesen war.

Noch ist zu bemerken, dals die Gase bei jeder Mblesung entweder vollkonmen trocken oder ganz mit Dampf gesätligt waren. Die Correctionen für die Temperalur wurden nach

so starke Explosionen verursachtc, dafs kein gewöhnlicher Eudiometer ihrer Heftigkeit widerstchen konnte. Sie wurde daher in einem 0,8m. langen Eudiometer ausgeführt und das Gas darin vor sciner Verbrennung mit circa seinem 26fachen Volum atmosphärischer Luft und dem 3fachen Volum Sauerstofl gemengt; auf diese Weise wurde die Heftigkeit der Explosion genı̈̈fsigt und, wie Kolbe gezeigt hat (diese Annalen Bd. LIX, S. 208), die Oxydation des Stickstoffs zu Salpetersäure verhindert; rciner Sauerstoff kann zu diesem Zwecke nicht angewandt werden, weil der nach der Verbrennung bleibende Ueberschufs zu grols seyn würde, um sich durch Explosion mit Wasserstoff bestimmen zu lassen, wenn man nicht cin Eudiometer von unbequemer Länge anwenden will. Allcin diese Verdiinnung kann nicht über cine gewisse Gränze hinausgeführt werden, obne die Genauiglieit der Resultate zu becinträchtigen, indem bei zu starker Verdünnung und dadurch verursachter Erniedrigung der Verbrennungstemperatur ein Theil des Kohlenstoffs statt zu Kohlensäure nur zu Kohlenoxyd oxydirt wird, wie folgende Versuche zeigen, in welchen das Gas mit verschiedenen Quantitäten almosphärischer laft und Sauerstoff explodirt wurde.

Gasvolumen Luft und Sauerstoffvol. Verzehrt. 0 . Gebildete $\mathrm{CO}_{2}$.

$\begin{array}{rrrrr}\text { I. } & 1 & 36,3 & 4,40 & 2,82 \\ \text { II. } & 1 & 32,4 & 5,28 & \mathbf{3 , 1 7} \\ \text { III. } & 1 & 28,8 & 5,36 & \mathbf{3 , 2 8} \\ \text { IV. } & 1 & 18,7 & 5,54 & \mathbf{3 , 2 6 .}\end{array}$

Bei den Versuchen I, und II. war die Explosion sehr schwach und die spätere Verbrennung mit Wasserstoff zeigte eine gröfsere Menge Stickstoff an, als in Gase und in der Luft enthalten war, welches Resultat nur von ciner unvollkommenen Verbrennung herrühren konnte; im Versuch IV. war die durch dic Explosion erzeugte llitze so grofs, dafs sich ein Theil dos Quecksilbers verflüchtigte und die Innenseite der Röhre mit einer schwarzen llaut überzog, wovon nachher der gröfste Theil in weifse Krystalle von salpetersaurem Quecksilberoxydul verwandelt wurde. 
Marchand's Tafeln berechnet, in welchen dor Ausdehnungscocfficient fïr jeden Centesimalgrad zu 0,003665 angenommen ist; zur Reduction der mit Feuchligkeit gesültigten Gase dienten Regnaull's Tafeln, und für die Ausdehnung durch Alkoholdämple wurden die von Schmidt gegebenen Zahlen gebraucht.

Der Gehalt des in der angeführten Weise aus dem kleineren Eudiometer in das gröfsere übergefüllten Gasvolumens an brennbaren Gasen ergiebt sich aus der erslen Ablesung des iilergefüllten Volumens, wenn man davon die durch Absorption der gleichen Gasmischung vermiltelst Alkohol im kleinen Eudiomeler ermiltelte Stickstoffmenge abzicht. Die drilte und fünfte Ablesung, nämlich die Bestimmmung des Gasvolumens nach Zulassung von Sauerstoff und atmosphärischer Luft einerseits, und des nach Absorption der Kohlensäure zurückbleibenden Volumens anderseils, nebst jener ersten Ablesung genügen, um daraus die Quantilät des verbrannten Saucrstoffs abzuleiten. Bezeichnen wir nämlich die aus der erslen Beobachtung nach Abzug des ermittelten geringen Stickstoffgehaltes sich ergebende Menge von brennbaren Gasen mit c, den Gehalt des durch die drilte Ablesung bestimmlen Gasvolumens an Sauerstoff und Sticksloff mit $(\mathrm{N}+0)$, ferner das gesammle beobachtele Gasvolumen der drillen Ablesung mit A, so folgt daraus die Gleichung :

$$
\mathrm{c}+(\mathrm{N}+0)=\mathrm{A} \text {. }
$$

Bezeichnen wir ferner das nach Absorption der Kohlensïure beibende Volumen der fünften $\Lambda$ blesung mit $B$, die fragliche verlirannte Sauerstoffmenge aber mit $x$, so ist, da B allen Stickstoff von $(\mathbf{N}+\mathbf{0})$, aber um so viel weniger Sauerstoff entlält, als durch die Verbrennung verzehrt ist,

$$
(\mathrm{N}+\mathrm{0})=\mathrm{B}+\mathrm{x} \text {. }
$$

Setzen wir daher für $(\mathrm{N}+0)$ den Werlh $\mathrm{B}+\mathrm{x}$ in obige Gleichung, so crhalten wir den Ausdruck :

$$
\mathrm{c}+\mathrm{B}+\mathrm{x}=\mathrm{A}
$$

und hieraus für $x$ den Werth : 


$$
\mathbf{x}=\mathbf{A}-\mathbf{c}-\mathbf{B} .
$$

Durch diese Berechnung ist man der Nolhwendigkeit überhoben, die Zusammenselzung der in's Eudiometer eingelassenen almosphürischen Luft zu berücksichtigen, wolurch man cinigen nicht unbedeutenden Fehlern entgeht.

Die Quantilät der gebildeten Kohlensäure wird leicht durch dic Diflerenz der vierten und fünften Ablesung gefunden.

Endlich zeigt die Verpuffung des nach Absorption der Kohlensäure gebliebenen Rückstandes mit einem Ueberschufs von Wasserstoff an, ob zuvor eine vollsländige Verbrennung der brennbaren Gase stallgefunden hat. In diesem Falle muls nämlich der aus den drei letzten Ablesungen sich ergebende Slicksloffgehalt sehr nahe mit dem in dem brennbaren Gase und der almosphärischen Luft eingeführten Slickstoff übereinstimmen.

Bei allen folgenden Versuchen war die durch Verbrennung mil überschüssigem Sauersloff entwickelle Wärme nie so slark, dafs sich Quecksill,er verflüchligle, oder dafs sich dabei auf Koslen des Stickstofls Salpelersäure bildete.

I.

Beobach- Temp. C. Diff. der Barome- Corr. Vol. tetes Vol. Quecksilber- ter bei $0^{\circ}$ C.u.

Angewandles Gas höhen $\quad 1_{m}$ Druek

(trocken) . . . . 116,2 7,9 $74,9 \mathrm{~mm} \quad 738,8 \mathrm{~mm} \quad 81,75$

Nach Absorplion durch

$\mathrm{SO}_{3}$ (trocken) . . $\quad 93,6 \quad 8,2^{\circ} \quad 30,5 \quad 735,1 \quad 64,03$

Nach Herausnahne eines

Theils zur Verbrennung (trocken). .

$82,3 \quad 9,0^{\circ} \quad 42,3 \quad 735,3 \quad 55,21$

Nach Alsorplion durch

Alkohol

$2,7 \quad 9,2^{\circ} \quad 42,8 \quad 732,9 \quad 1,76$

II.

Angewandtes Gas

$\begin{array}{llllll}\text { (lrocken) . . . . } 123,1 & 8,3^{\circ} & 6,1 & 741,3 & 87,83\end{array}$ 
Nach Absorption durch

Beobach- Temp.C. Diff, der Barome- Corr. Vol. tetes Vol. Quccksilber- ter bei $0^{\circ} \mathrm{C}$. u. höhen 1m Druck

$\mathrm{SO}_{3}$ (Irocken) . . 98,8 7,70 24,0mm 739,4mm 68,74 Nach Herausnahne eines

Theils zur Verbrennung (trocken) . . $\quad 87,8 \quad 8,2^{\circ} \quad 37,3 \quad 733,1 \quad 59,31$

Nach Absorption durch

Alkohol $\quad \cdot \quad \cdot \quad \cdot \quad \cdot \quad 2,9 \quad 8,1^{0} \quad 56,5 \quad 734,8 \quad 1,86$

Angewandles Gas

III.

$\begin{array}{llllllll}\text { (feucht) } & . & . & 102,3 & 8,4^{0} & 618,8 & 735,7 & 10,79\end{array}$

Nach Zulassung von

alm. Luft (trocken) . $\quad 543,7 \quad 8,6^{\circ} \quad 160,8 \quad 735,6 \quad 298,60$

Nach Zulassung von

Sauerstoff (feucht) • $\quad 569,1 \quad 8,7^{\circ} \quad 137,8 \quad 735,6 \quad 325,07$

Nach der Verbrenuung

(feuclit) . . . . $\quad \begin{array}{ccccc}537,5 & 8,7^{\circ} & 166,7 & 735,6 & 291,96\end{array}$

Nach Absorplion der

Kohlensäure (Irocken) 498,0 $\quad 8,6^{\circ} \quad 202,7 \quad 735,8 \quad 257,37$

Nach Zulassung von

Wasserstoff (trocken) $\quad 626,9 \quad 9,0^{\circ} \quad 87,2 \quad 735,6 \quad 393,51$

Nach der Verbrennung

(feucht) . . . . $549,6 \quad 9,0^{\circ} \quad 155,4 \quad 735,3 \quad 303,96$

Angewandtes Gas

IV.

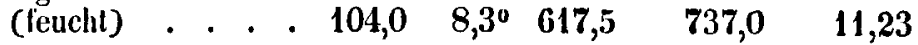

Nach Zulassung von

atm. Luft (feucht) . $\quad 538,3 \quad 8,3^{\circ} \quad 166,0 \quad 736,8 \quad 293,91$

Nach Zulassung von

$\begin{array}{llllll}\text { Sauersloff (Feucht) } & 577,0 & 8,0^{\circ} & 131,6 & 736,1 & 334,38\end{array}$

Nach der Verbrennung

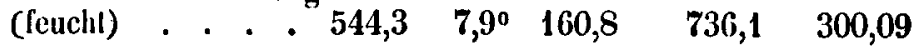

Nach Absorption der

Kohlensäure (trocken) 505,2 $\quad \mathbf{7 , 6}^{\circ} \quad \mathbf{1 9 6 , 4} \quad \mathbf{7 3 3 , 5} \quad \mathbf{2 6 3 , 9 9}$

Nach Zulassung von

Wasserstoff (trocken) $\mathbf{6 3 6 , 5} \quad 8,3^{\circ} \quad \mathbf{7 9 , 1} \quad \mathbf{7 3 3 , 1} \quad 403,98$

Nach der Verbrcunung

(fenchl) . . . . $532,0 \quad 8,3^{n} \quad 171,8 \quad 732,2 \quad 285,10$ 
Den Versuchen I und II zufolge enthült die urspriingliche Gasmischung in 100 Theilen :

I. II. Mittel

Durch Schwefelsäure alssorbirbares Gas $\quad 21,68 \quad 21,73 \quad 21,70$

Brenubares, nicht durch Schwefelsiiture

absorbirbares Gas. . . . . . . $75,82 \quad 75,82 \quad 75,82$

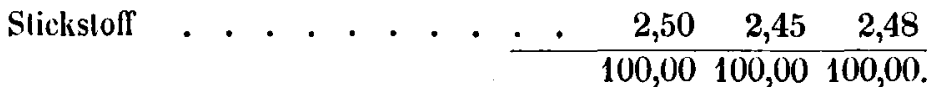

Aus den Analysen III. und IV. ergeben sich für die von 1 Vol. des brembaren Gases verzehrle Sauerstoffmenge und die dadurch gebildete Kohlensïure folgende Werthe :

III. IV.

$\begin{array}{lll}\text { Verzehrler Saucrsioff } & \mathbf{5 , 4 8} & \mathbf{5 , 4 7} \\ \text { Gebildete Kohlensiurc } & \mathbf{3 , 3 1} & \mathbf{3 , 3 2} .\end{array}$

Wenn man in Erwïgung zichl, dafs die gasförmigen Körper sich immer nur in den einfachsten Verhiiltnissen mil einander verbinden, so kann man nicht in Zweifel dariber seyn, dafs man es hier nicht mit einem einzelnen Gase, sondern vielmehr mil einem Gasgremenge zu thun hat. Zugleich ist es sehr unwahrscheinlich, dafs ein Gas von der allgemeinen Formel $\mathrm{C}_{n} H_{n}$ nach der Einwirkung ranchender Schwefolsäure vorhanden sey, da alle Gase dieser Reilie, so weit bis jelzt bekannt ist, von jener Säure schnell absorbirt werden. Da aufserdem die Abwesenheit von Wasserstoff und Grubengas durch dic Löslichkeit jenes Gases in Alkohol erwiesen ist, so gewinnt die Vermuthung einigen Raum, dafs die Mischung aus Melhyl und dem bisher nicht isolirten Radicale Aelhyl bestehe. Inwieweit die erhaltenen Resultate der eudiometrischen Analyse und der Bestimmung des specifischen Gewichts mit dieser Vermulhung uibereinstimmen, wird sich aus den nachfolgenden Betrachtungen ergeben. Bei der Bestimmung der relativen Mengen des Melhyls und Acthylgases ist in Betracht zu ziehen, dafs Aethyl sein $6 \frac{1}{2}$ faches Volum Sauersloff zur Verbrennung bedarf, und damit sein 4faches Volum Kohlensäure erzeugt, während Melhyl $3 \frac{1}{2}$ Volumina 
Sauerstoff erfordert und 2 Volumina Kohlensäure liefert; da das Volum der gemischten Gase bekannl ist, so bedarf es zur Bildung zweier Gleichungen nur noch einer bekannten Gröfse, um daraus die relativen Mengen der beiden Gase abzuleiten; ich habe dazu die durch die Verbrennung mit Sauerstoff verursachle Volumvern̄inderung benulzt, welche durch Subtraction der vierten Ablesung von der dritten (Versuch III. und IV.) erhalten wird, ein Werth, in welchem zugleich der verzehrte Sauerstoff und die gebildete Kohlensäure zusammengefafst sind; da diese Volumverminderung durch den verschwundenen Sauerstoff und das Volumen des zu Kohlensäure und Wasser verbrannten brennbaren Gases, weniger das Volumen der gebildeten Kohlensäure ausgemacht wird, so erhellt, dafs das Aethyl für sich eine seinem $3 \frac{1}{2}$ fachen Volum, und Methyl seinerseits eine seinem $2 \frac{1}{2}$ fachen Volum gleiche Zusammenzichung bewirkt. Wenn wir daher das Volum des bremnbaren Gases (erhalten durch Subtraction der bekannten Menge Stickstoff vom beobachtelen Volum) durch A bezeiclınen, die durch die Verbrennung erfolgte Contraction $B$, und die unbekannten Mengen des Aethyls und Methyls beziehungsweise $x$ und $y$ nennen, so haben wir folgende Gleichungen :

$$
\begin{aligned}
x+y & =A \\
7 x+5 & =B,
\end{aligned}
$$

woraus sich für $\mathrm{x}$ und $\mathrm{y}$ folgende Werthe ergeben :

$$
\begin{aligned}
& x=\frac{2 B-5 A}{2} \\
& y=\frac{7 A-2 B}{2}
\end{aligned}
$$

Der Analyse III. zufolge bedurften 10,79 Vol., aus 0,34 Vol. Stickstoff und 10,45 Vol. brennbarer Gase besteliend, 57,25 Vol. Sauerstoff zur Verbrennung und bildelen 34,59 Vol. Kohlensäure; die Volumverminderung betrug daher 33,11 Vol. Durch 
Substitulion der Zahlen 10,45 und 33,11 für $\Lambda$ und $B$ in obige Gleichungen erbält man :

$$
\begin{aligned}
& x=6,98 \\
& y=3,47 .
\end{aligned}
$$

In der Analyse IV. nahmen 11,23 Vol, zusammengeselat aus 0,35 Vol. Stickstoff und 10,88 Vol. lorennbarer Gase, 59,51 Vol. Sauersloff auf und lieferten 36,10 Vol. Kohlensäure. Die Volumverminderung betrug daher 34,29 Vol. Daraus ergeben sich für $x$ und $y$ die Werthe :

$$
\begin{aligned}
& x=7,09 \\
& y=3,79 .
\end{aligned}
$$

Demnach würden die $75,82 \mathrm{pC}$. der durch rauchende Schwefelsäure nicht absorbirten Gase bestehen aus

$$
\text { III. IV. Mittel }
$$

\begin{tabular}{lrrr} 
Aethyl & $\mathbf{5 0 , 6 4}$ & $\mathbf{4 9 , 4 1}$ & $\mathbf{5 0 , 0 3}$ \\
Methyl & $\mathbf{2 5 , 1 8}$ & $\mathbf{2 6 , 4 1}$ & $\mathbf{2 5 , 7 9}$ \\
\hline & $\mathbf{7 5 , 8 2}$ & $\mathbf{7 5 , 8 2}$ & $\mathbf{7 5 , 8 2}$.
\end{tabular}

Un die Zusammensetzung und das Condensationsverhaillnifs des durch rauchende Schwefelsäure absorbirten Gases zu ermitleln, wurde das urspriingliche dieses Gas enthaltende Gemenge, unter Berücksichligung aller der zuvor beobachteten Vorsichtsmafsregeln, mit almosphärischer Luft und Sauerstolf verpufft. Es wurden folgende Zahlen erhallen :

$$
\text { V. }
$$

Beobachte- Temp.C. Diff. der Barome- Corr.Vol. tes Yol. Quecksilber- ter. bei $0^{\circ} \mathrm{C}$. und

Angewandtes Gas hülıen 1 mi'uck

(feucht) . . . . 99,6 7,4 $621,5 \mathrm{~mm} \quad 735,9 \mathrm{~mm} \quad 10,35$

Nach Zulassung von

$\begin{array}{llllll}\text { alm. Luft (feucht) } \quad .508,9 & 7,2^{\circ} & 192,4 & 735,8 & \mathbf{2 6 5 , 6 0}\end{array}$

Nach Zulassung von

$\begin{array}{lllll}\text { Sauerstoff (feucht) . 559,5 } \quad 7,2^{\circ} & 146,0 & \mathbf{7 3 5 , 8} & \mathbf{3 1 7 , 3 7}\end{array}$

Nach d. Verbrennung

(feucht) . . . . 530,8 $7,1^{\circ} \quad 172,0 \quad 736,1 \quad 287,95$ 
Beobaclt- Temp.C. Diff. der Barome- Corr. Vol. tetes Vol.

Quecksilher- ter hei $0^{\prime \prime} \mathrm{C}$. u.

Nach Absorption der tülien $1 \mathrm{~m}$ Druck

Kohlensäure(trocken) $\quad 494,3 \quad 7,3^{\circ} \quad \mathbf{2 0 6 , 8 m m} \quad \mathbf{7 4 1 , 3 \mathrm { mm }} \mathbf{2 5 7 , 3 2}$

Nach Zulassung von

Wasserstoff (trocken) $701,3 \quad 7,3^{\circ} \quad \mathbf{2 4 , 1} \quad \mathbf{7 4 1 , 2} \quad 489,80$

Nach der Verbrennung

(feuchl) . . . . $\quad .563,3 \quad 7,3^{\circ} \quad 142,8 \quad 741,5 \quad 324,29$

VI.

Angewandtes Gas

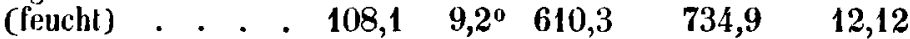

Nach Zulassung von

atm. Luft (feucht) . $\quad 533,7 \quad 9,2^{\circ} \quad 167,5 \quad 734,9 \quad 288,45$

Nach Zulassung von

$\begin{array}{llllll}\text { Sauerstoff (feucht) } \quad 589,9 & \mathbf{9 , 0 ^ { \circ }} & \mathbf{1 1 7 , 1} & \mathbf{7 3 4 , 2} & \mathbf{3 4 7 , 4 9}\end{array}$

Nach der Verbrennung

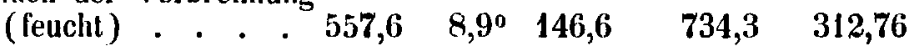

Nach Absorplion von

Kohlensäure (trocken) 518,7 $\quad 9,0^{\circ} \quad 181,2 \quad 733,1 \quad 277,13$

Nach Zulassung von

Wasserstoff ('rocken) 706,3 $\quad \mathbf{9 , 0}^{\circ} \quad \mathbf{1 8 , 2} \quad \mathbf{7 3 2 , 6} \quad \mathbf{4 8 8 , 4 7}$

Nach der Verbrennung

(feucht) . . . . $558,0 \quad 8,8^{\circ} \quad 146,4 \quad 732,4 \quad 312,18$

Nach der 'Analyse V. verzehrlen 10,35 Volumina, 10,10

Vol. brennbare Gase enthallend, 49,95 Vol. Sauersloff und bildelen 30,63 Vol. Kohlensäure; 10,35 Vol. des ursprünglichen Gases enthalten aber dem arithmelischen Miltel der Analysen l. und II. zufolge 7,85 Volumina brennbares, durch Schwefelsäure nicht absorbirles Gas, welches (vid. Analysen Ill. und IV.) zu seiner Verbrennung 43,02 Vol. Sauersloff verlangt und 26,06 Vol. Kohlensïure bildet; es bleiben daher für die 2,25 Vol, des durch Schwefelsïure absorbirten Gases 6,93 Vol. vom verzehrten Sauersloff und 4,57 Vol. von der gebildeten Kolılensäure übrig.

Die Analyse VI. führt zu einem ähnlichen Resultal; 12,12 Volumina, aus 11,82 Volumina brennbarer Gase bestehend, nahmen 58,54 Vol. Sauerstoff auf und gahen 35,63 Vol. Kohlen- 
säure; und da die Gasgemenge, unserer Annahme gemäfs, 9,19 Vol. der Mischung von Aethyl und Melhyl, welche 50,36 Vol. Sauerstoff brauchen und 30,51 Vol. Kolılensäıre bilden, enthält, so kommen auf die ührighleibenden, durch Schwefelsäure absorbirten 2,63 Gasvolumina 8,29 Vol. Sauerstofl und 5,00 Vol. Kohlensäure.

Ein Volumen des durch Schwefelsäure absorbirbaren Gases steht daher zu dem bei seiner Verbrennung consumirten Sauerstoff und zur erzeugten Kohlensäure in folgendem Verhälınisse:

$\begin{array}{lccc} & \text { V. } & \text { Vr. } & \text { Mittel } \\ \text { Verzehrler Sauerstoff } & 3,08 & 3,11 & 3,09 \\ \text { Gebildele Kohlensäure } & 2,03 & 1,95 & 1,99 .\end{array}$

Das fragliche Gas scheint somit ganz die Zusammensetzung und das Condensationsverhältnifs des Elayls zu besitzen, von welchem 1 Vol. zur Verlirennung 3 Vol. Sauerstoff bedarf und 2 Vol. Kohlensäure erzengt : Zahlen, welche mit den gefundenen hinreichend übereinstimmen, wenn man bedenkt, dafs etwaige Fehler in den Beobachlungen sich auf einen kleinen Theil des brennbaren Gases concentriren.

Zur Controle dieser Analysen wurde das mil Chlorcalcium vollständig getrocknete Gas $\alpha$ durch einen, mit Antimonsuperchlorid gefüllten Kaliapparat geleitel, wodurch das von Schwefelsäure absorbirbare Gas vollständig und schnell verschluckl wird, während Aelhyl, wie ich späler zeigen werde, und Methyl keine Veränderung erleiden. Eine unbestimmte Menge dieser so erhaltenen bräunlichen krystallisirbaren Fliissigkeit wurde mit Kupferoxyd und vorgelegtem metallischem Kupfer langsam verbrannt. Nach der Verbrennung hatte sich das Gewicht des Kaliapparates un 0,1164 Grm. und das des Chlorcalciumrohrs um $0,0473 \mathrm{Grm}$. vermehrt.

Aus diesen Zahlen ergiebt sich das Verhältnifs der Kohlenstoffäquivalente zu den Wasserstoffäquivalenten wie folgt : 


$$
\begin{aligned}
& \mathrm{C}: \mathrm{H}=0,0053: 0,0053 \\
& \text { oder } \mathrm{C}: \mathrm{H}=2: 2
\end{aligned}
$$

ein Resultat, welches über die Zusammenselzung des durch Schwefelsäure absorbirbaren Gases keinen Zweifel läfs?. ob dicser Körper wirklich mit Elayl identisch ist, ist durch weitere Untersuchungen zu ermilteln.

Die procentische Zusammenselzung des Gases $\alpha$ kann, den mitlleren Resultaten der angefühten Analysen nach, folgendermafsen ausgedrückl werden :

\begin{tabular}{lr} 
Aethyl & 50,03 \\
Methyl & 25,79 \\
Elayl & $\mathbf{2 1 , 7 0}$ \\
Stickstoff & 2,48 \\
\cline { 2 - 2 } & $\mathbf{1 0 0 , 0 0}$
\end{tabular}

Das specifische Gewicht ciner so zusammengesetzlen $\mathrm{Mi}$ schung stimnt mil dem durch den Versuch (S. 178) gefundenen 1,5250 nahe überein, wie man aus nachslehender einfacher Berechnung ersieht :

$$
\begin{gathered}
\mathrm{C}_{4} \mathrm{H}_{3}=50,03 \times 2,00390=100,2551 \\
\mathrm{C}_{2} \mathrm{H}_{3}=25,79 \times 1,03652=26,7319 \\
\mathrm{C}_{2} \mathrm{H}_{2}=21,70 \times 0,96742=20,9930 \\
\mathrm{~N}=2,48 \times 0,96740=\frac{2,3992}{150,3792} \\
\quad=100,00 \quad 1,503792 .
\end{gathered}
$$

Untersuchung des Gases $\not \beta$.

Dieses Gas, dessen Volumen etwa nur ío vom Volumen des vorigen betrug, wurde auf dieselbe Weise wie jenes untersucht; dic eudiometrische Analyse gab folgende Zahlen :

Beobach- Tem, C. Diff, der Barome- Corr. Vol. tetes Vol. Quccksilber- ter bei $0^{\circ} \mathrm{C}$. II.

Angewandtes Gas höhen $\quad$ im Druck $^{\text {m }}$

(trocken) . . . . 160,6 13,20 4,3mm 737,8mm 112,37 Nach Absorption durch

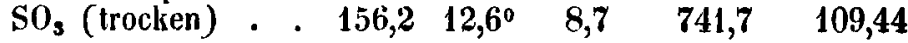


Nach Herausnahme eines

Beobach- Temp. C. Dilf. der Barome- Corr. Vol. tetes Vol. Quecksilber- ter bei $0^{\circ} \mathrm{C} . \mathrm{u}$.

Theils zur Verbrennung

(trocken) . . . . $128,8 \quad 13,9^{\circ} \quad 37,8 \mathrm{~mm} \quad 742,1 \mathrm{~mm} \quad 86,32$

Nach Absorption durch

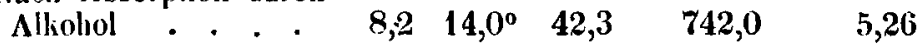

Das untersuchte Gas enthielt daher in 100 Theilen :

Durch Schwefelsäure absorbirbares Gas . . . 2,61

Brennbares, nicht durch $\mathrm{SO}_{3}$ absorbirbares Gas . 91,46

Stickstoff . . . . . . . . . . . . . . 5,93

100,00 .

Bei der Verbrennung des, nach beendeter Absorption durch Schwefelsäure, in's gröfsere Eudiomeler zuvor übergefüllten Theils des Gases mil Sauerstoff wurden folgende Zahlen erhalten. :

II.

Beobach- Temp. C. Diff. der Barome- Coir. Vol. tetes Vol. Quecksilber- ter bei $0^{\circ}$ C. II.

Angewandes Gas

höhen $1 \mathrm{~m}$ Druck

(fieuclit) . . . . $116,9 \quad 13,5^{\circ} \quad 598,9 \mathrm{~mm} \quad 741,8 \mathrm{~mm} \quad 14,64$

Nach Zulassung von

$\begin{array}{lllllll}\text { Luft (feucht) } & \text { - } & 454,3 & 13,4^{\circ} & 240,8 & 741,8 & 211,97\end{array}$

Nacl Zulassung von

Sauerstoff (feuchi) • $540,2 \quad 13.5^{\circ} \quad 160,5 \quad 741,7 \quad 293,25$

Nach der Verbreunung

(feucht) . . . . 502,0 13,40 195,9 741,8 255,71

Nach Absorption der

Kohlensäure (trocken) 463,8 $\quad 13,3^{\circ} \quad 236,6 \quad 738,5 \quad 221,96$

Nach Zulassung von

$\begin{array}{lllll}\text { Wasserstoff (trocken) } & \mathbf{7 1 6 , 8} & \mathbf{1 3 , 3 ^ { \circ }} \quad \mathbf{1 3 , 7} & \mathbf{7 3 8 , 4} & \mathbf{4 9 5 , 3 3}\end{array}$

Nach der Verbrennung

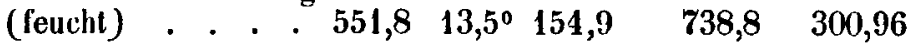

III.

Angewandles Gas

$\begin{array}{llllll}\text { (feucht) . . . } & 121,2 \quad 13,6^{\circ} & 594,3 & 738,7 & 15,33\end{array}$ 
Beobach- Temp.C. Diff. der Barome- Corr. Vol. tetes Vol. Quecksilber- ter bei $0^{n} \mathrm{C}$. u. höhen 1m Druck

Nach Zulassung von

Lult (feuchl) . . . 448,0 13,7॰ 247,7 $\mathrm{mm} \quad 738,6 \mathrm{~mm} \quad 204,43$

Nach Zulassung von

Saucrsioff (feuclit) . $\quad 529,4 \quad 14,0^{\circ} \quad 171,2 \quad 739,3 \quad 280,08$

Narh der Verbrennung

(feucht) . . . 488,0 14,30 209,4 $739,8 \quad 240,34$

Nach Absorption der

Kohlensäure (trocken) 440,9 $13,5^{\circ} \quad 254,7 \quad 742,0 \quad 204,72$

Nach Zulassung von

Wasserstolf (trocken) $662,7 \quad 13,9^{\circ} \quad 52,2 \quad 742,1 \quad 435,04$

Nach der Verhrennung

(feucht) . . . . 520,6 14,0 $178,0 \quad 742,2 \quad 273,49$

Der Analyse II. zufolge erforderten 14,64 Volumina, welche (naclı Analyse 1.) 13,75 Vol. lrennbares Gas enthalten, 57,54 Vol. Sauerstoff zur Verbremungr und erzenglen damit 33,75 Vol. Kohlensäure. Die durch die Verbrennung hervorgebrachle Volumverminderung belrug 37,54 Vol. Nach der Analyse III. verlirannten 15,33 Vol. (14,40 Vol, bremulares Gas enlhaltend) mit 60,96 Vol. Sauerstuff zu 35,62 Vol. Kohlensäure. Die eintrelende Volunverminderung war gleich 39,74 Vol. Diesen Zahlen entsprechen folgende Verhältnisse :

Vol. des brennb. Gases Verbr. Sauerstoff Erzeugte Kohlensäure

$\begin{array}{ccccccc}\text { Analyse } & \text { II. } & 1 & : & 4,18 & : & 2,46 \\ " & \text { III. } & 1 & : & 4,23 & : & 2,47 .\end{array}$

Diese Zahlenwerthe in Verbindung mit der beobachteten Alsorbirbarkeit cines Theils des Gases durch rauchende Schwefelsäure lassen es unzweifelhaft erscheinen, dals das Gas $\beta$ diesellen Bestandthcile enthält, wic das Gas $\alpha$; nur in einem andern Verhältnissc. Bei Anwendung der obigen Formeln auf die Resultate der Analysen II. und III. ergeben sich für $x$ und $y$ folgende Werthe : 


$$
\begin{gathered}
\text { Il. } \\
x=3,17 \\
x=10,58 . \\
\text { III. } \\
x=3,74 \\
y=10,66 .
\end{gathered}
$$

Der procentischen Zusammensetzung jenes Gasgemenges entsprechen demnach folgende Zahlen, wenn man den Stickstoffgehalt desselben umberücksichligt lärst :

\begin{tabular}{lrc} 
Elayl & II. & III. \\
Aethyl & $\mathbf{2 , 7 8}$ & 7 \\
Methyl & $\mathbf{2 2 , 4 1}$ & $\mathbf{7 1 , 9 7}$ \\
\cline { 1 - 1 } & $\mathbf{2 5 , 2 5}$
\end{tabular}

Wenn man den in der Glasröhre enthallenen krystallinischen Körper, unuillelbar nachdem die Entwickelung des Gases $\alpha$ aufgehört hat, mit Wasser übergiefst, anstalt ihn, wie es oben geschehen ist, vorher 12 Stunden lang sich selhst zu ül,erlassen, so erhält man ein Gasgemenge, dessen Zusammenselzung durch das Verhältnifs scines Gehalts an Aethyl und Melhyl von der des anderen schr abweicht, wie sich aus der nachfolgenden eudiometrischen Verbrennung des Gases crgiebt, welches, so bereilel und unmitlelbar über Quecksilber aufgefangen, zuvor durch eine mit rauchender Schwefelsäure getränkte Koakkugel vom Elayl und' den beigemengten Dämpfen von Jodäthyl und von der hierbei entwickelten schwefligen Säure durch eine Kalikugel befreit war. Das Gas wurde von Alkohol vollständig absorbirt und war daher stickstoffrei :

$$
\begin{gathered}
\text { Beobach- Temp. C. Diff. der Barome- Corr. Vol. } \\
\text { Quecksilber- } \\
\text { tetes Vol. } \\
\text { höhen }
\end{gathered} \quad \begin{aligned}
& \text { bei } 0^{\circ} \mathrm{C} .11 . \\
& 1 \mathrm{~m} \text { Druck }
\end{aligned}
$$

Angewandtes Gas

(feucht) . . . . $139,0 \quad 8,3^{\circ} \quad 577,5 \mathrm{~mm} \quad 732,2 \mathrm{~mm} \quad 19,76$ Nach Zulassung von
Luft (feucht) .
487,9
$8,3^{\circ} 213,2$
731,5
241,53 
Beobach- Temp.C. Diff. der Baromc- Corr. Vol. tetes Vol. Quecksilber- ter bei $0^{\circ} \mathrm{C}$. u.

Nach Zulassung von

Sauerstoff (feuchl) ～$\quad 558,6 \quad 8,4^{\circ} \quad 142,8 \mathrm{~mm} \quad 731,2 \mathrm{~mm} \quad 314,42$

Nach der Verbrennung

(feuchl) : . . . $502,9 \quad 8,3^{\circ} \quad 199,2 \quad 731,0 \quad 255,55$

Nach Absorption der

Kohlensäure (trocken) $\quad 435,8 \quad 8,2^{\circ} \quad 260,5 \quad 733,1 \quad 199,95$

Die Zahlen führen zu dem Verhällnifs von :

$$
x: y=9,47: 10,29
$$

oder zu folgender procentischen Zusammenselzung :

Aethyl $\quad 47,93$

Melhyl $\frac{52,07}{100,00}$

Hiernach gewinnt die Vorslellung Wahrscheinlichkeit, dafs das so leicht condénsirbarc Aethyl nur als mechanisch beigemengle Fliissigkeit vorhanden ist, und bei gewöhnlichem Atmosphärendruck nach und nach von den Kryslallen abdunstel, das Melhyl aber, dessen Menge unter denselben Verhältnissen nicht abnimmt, sich in chemischer Verbindung mit den Krystallen betindet. Letzlere würden daher ein selir geeigneles Miltel darbieten, um andere Körper auf Methyl im Status nascens einwirken zu lassen.

Die Zerlegung des Jodälhyls durch Zink läfst sich, den oligen Thatsachen zufolge, durch folgende einfache Gleichung ausdrüicken :

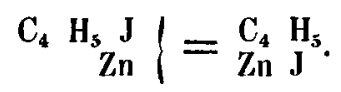

Von den in Freiheil gesetzten Aethyl wird aher gleichzeitig ein Theil in gleiche Volumina Elayl und Methyl zersetzt :

$$
\mathrm{C}_{4} \mathrm{H}_{5}=\mathrm{C}_{2} \mathrm{H}_{2}+\mathrm{C}_{2} \mathrm{H}_{3} \text {, }
$$

wohei ein Theil des letzleren sich wahrscheinlich mit dem Jodzink in einem ganz bestimmlen Atomverhältnifs zu der obigen krystallinischen Verlbindung vereinigt. Die Analysen des 
Gases $\alpha$ weisen darin einen kleinen Uebersthuls an Methyl iiber den Gehalt an Elayl nach, wchcher noch elwas gröfser ausgefallen seyn wiirde, wenn man dic Gase $\alpha$ und $\beta$ in demselben Behälter aufgefangen liälc : diescr Ueberschufs rührt wahrscheinlich von eimer Spur von Feudhligkcit her, welche entweder dem Jodälhyl oder dem Zink anhingr, da, wie wir weiter unten schen wcrden, das Jodäthyl mit Zink bei Gegentwart von Wasser in Zinkoxyjodid und zwei Volumina Methylgas zerlegl wird.

Wenn man erwägt, dal's das Methyl und Elayl aller Wahrscheinlichkeil nach einen nicderern Kochpunkt Jaben, als das Aethyl, so mufs die Vermullumg Raum gewinnen, dafs beim Oeffnen des Zersetzungsrohrs zucrst dic beiden ersteren enlweichen, und dafs dasjenige Gas, welches man beim Verdanpfen der lelzten Antheile der condensirten Flüssigkeit erhält, reines Aelhyl sey. Um hierüber Gewifsheit zu erhalten, wurden zwei Röhren auf die oben heschriebene Weise mit Jodälhyl und Zink gefüllt, sodann hermelisch verschlossen und in Oelbade erhitzl. Das nach dem Abbrechen des äufsersten Endes ihrer capillaren Spitzen unter Quecksilber cntslrömende Gas wurde, nachdem der gröfste Theil ausgetrelen war, erst dann in eincr Glocke über Quecksilber aufgefangen, als dasselbe anfing, in einem langsamen regelmiilsigen Strome zu cntweichen. Beide Röhren lieferten clwa 180 Cbc. Es wurde alsdann von dem etwa beigemengten Elayl und den Dämpfen von Jodälhyl durch rauchende Schwefelsäure befrcil und dic dabei cntbundenc Sclıwefelsäure nachträglich durch cine Kalikugel absorbirt.

Zwei von dem so dargestcllten und gereiniglen Gase angestellte Analysen gaben folgende Dalen : 
I.

Beobach- Temp.C. Diff. der Barome- Corr. Vol. tetes Vol. Quecksilber- ter hei $0^{\circ} \mathrm{C}$. u.

Angewandles Gas höhen

Im Druck

(feuchi) . . . . $90,0 \quad 12,6^{\circ} \quad 626,0 \mathrm{~mm} \quad 751,1 \mathrm{~mm} \quad 9,82$

Nach Zulassung von

Luft (feucht) . . . $\quad 475,9 \quad 12,6^{\circ} 216,4 \quad 751,2 \quad 238,32$

Nach Zulassung von

$\begin{array}{lllll}\text { Sauerstoff (feucht) . } \quad 520,7 & 12,6^{\circ} & 174,9 & 751,7 & 281,66\end{array}$

Nach der Verbrennung

(feuchl) . . . . 484,8 $12,5^{\circ} 208,0 \quad 752,0 \quad 247,17$

Nach $\Lambda$ bsorption von

Kohlensäure (lrocken) $436,1 \quad 11,7^{\circ} \quad 255,0 \quad 753,3 \quad 208,37$

Nach Zulassung von

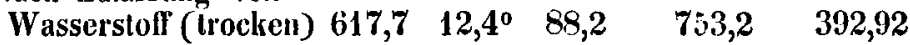

Nach der Verlsrennung

(feucht) . . . $548,2 \quad 12,6^{\prime \prime} 148,4 \quad 752,8 \quad 311,00$

Il.

Angewandtes Gas

(feucht) . . . . $\quad 91,8 \quad 12,8^{\circ} \quad 623,1 \quad 752,7 \quad 10,40$

Nach Zulassung von

Luft (feucht) . . . $471,2 \quad 13,0^{\circ} \quad 219,9 \quad 752,6 \quad 234,56$

Nach Zulassung run

Sauerstoff (feucht) . $535,1 \quad 12,9^{\circ} \quad 160,6 \quad 751,7 \quad 296,35$

Nach der Verbrenmung

(feucht) . . . . $498,8 \quad 12,8^{\circ} \quad 194,0 \quad 751,1 \quad 260,19$

Nach Absorption der

Kohlensäure (Irocken) $454,3 \quad 13,0^{\circ} \quad 237,2 \quad 741,5 \quad 218,69$

Nach Zulassung von

Wasserstoff (Irocken) $\quad 644,7 \quad 13,1^{\circ} \quad 63,9 \quad 740,8 \quad 416,41$

Nach der Verbrennung

(feucht) . . . . $532,7 \quad 13,0^{\circ} \quad 151,8 \quad 740,0 \quad 293,39$

Hieraus wird folgendes Verbältuifs des untersuchten brennbaren Gases zu dem verzehrten Sauersioff und der erzeugten Kohlensäure bereclnnct : 


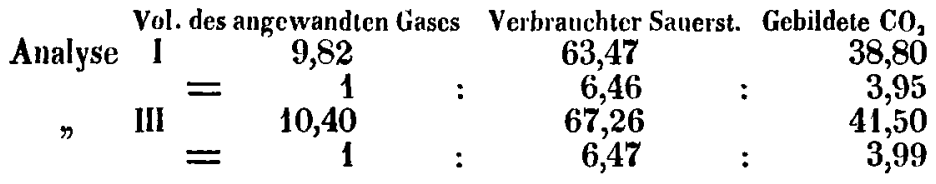

Das Gas besleht daher aus reinem Aelhyl, welches der Theorie gemäls das 6,5fache seines Volumens an Sauerstoff zur Verbrennung bedarf und sein 4faches Volumen Kohlensäure liefert, mit welchen Zahlen die.durch dic Analyse gefundenen nahe übcreinstimmen.

Obschon obige Resultate der. Analyse die Identität jenes Gases mit dem Aelhyl kaum lezwcifeln lassen, so hielt ich es doch nicht für uiberflüssig, mich noch durch einen besondern Versuch davon zu überzeugen, dafs es nicht elwa ein Gemenge, sondern ein wirklich einfaches Gas sey. Ich unterwarf es zu diesem Zwecke in der nachstehend beschriebenen Weise der Diffusion; die erhaltenen Dalen gewährten zugleich eine Controle fiir die friiher gcmachte Bestimmung seines specifischen Gewichtes. Einc 10 Zoll lange und $\underset{4}{3}$ Zoll weilc Glasröhre A, Fig. III, welche mil auf die Glaswand cingeätzter Millimeterscale versehen war, wurde mit einem 5 mm! dicken Gypspfropfen (b)

Fig. III. verschlossell. Sie war an diesem Ende aufserdem

$\underset{c}{\longrightarrow}$ eben geschliffen, so dafs sic durch eine mit Fett bestrichene Glasplalle (c) luftdicht verschlossen werden konnte, welche, wenn sic auflag, die äulsere Fläche des Gypspfropfens beinahe berührte. Das Instrument war zuvor auf die gewöhnliche Weise genau calibrirt und der Gypspfropfen möglichst vollkommen getrocknet. Un dic Röhrc mit Quecksilber und dann mit Gas zu füllen, wurde sie oben durch die Glasplatte fest verschlossen, darauf ein Arm eines dünnen Hebers in das untere Ende derselben bis dicht unter die Gypsfläche gebracht und so in ein mil Quccksilber gefülltes Gefuifs eingetaucht, bis das Metall, die durch den Heber ausströmende Luft langsam verdrängend, cben den Gypspfropfen berührle. 
Bei diesem Verfahren bleiben die Poren des Gypses offen, was nicht der Fall seyn würde, wenn das Quecksilber auf die gewöhnliche Weise in die Röhre eingefüllt wäre. Der Heber wurde darauf entfernt, sodann das zuvor von aller Feuchtigkeit volikommen befreite Gas durch die untere offene Mündung eingefüllt und scin Volumen mit Beobachtung aller nöthigen Vorsichtsmaalsregeln durch Ablesung an der Millimeterscale bestimmt. Nachdem alsdann die Röhre vermillelst eines in verticaler Richtung beweglichen Halters so gestellt war, dafs das Quecksilber inwendig und auswendig gleich hoch sland, wurde die aufliegende Glasplatte entfernt, worauf die Diffusion der äufseren atmosphärischen Luft und des eingeschlossenen Gases begann. Das Volumen des Gases nahm dabei allmälig zu, in demselben Maafse wurde die Rölıre, um das eingeschlossene Gas unter einem gleichen Druck der Atmosphäre zu erhalten, durch den Haller hinaufgeschoben, so dafs der äufsere und innere Quecksilberspiegel möglichst genau im Niveau blieben. Der Versuch wurde nach zehn Minulen lang staltgehabter Diffusion, durch Auflegen der Glasplatte wie vorher, unterbrochen, das Gas darauf durch eine Kalikugel sorgfältig getrocknet und sein Volumen wiederum gemessen. Es wurde dann in zwei Theile getheilt, die eine Hilfte in ein kurzes Eudiometer gebracht, um darin durch Absorplion vermittelst Alkohols den Gelıalt an alınosphärischer Luft zu beslimmen, und die andere Hälfte iı eineın gröfseren Eudiometer mit Luft und Sauerstoff verbrannt. Die zur richtigen Verbrennung erforderliche Menge der beillen letzleren Gase läfst sich leicht berechnen, nachdem man den Gehalt der Mischung an brennbarem Gas und atmosphärischer Luft durch Behandlung derselben in kleinen Eudiometer mit Alkohol ermittelt hat.

Beim Diffusionsversuch wurden folgende Zahlen abgelesen : 
Angewandtes Gas

$$
\text { I. }
$$

Beobach- Temp, C. Ditr. der Barome- Corr. Vol. tetes Vol, Quecksilber- ter bei $0^{\circ} \mathrm{C}$. u.

(lrocken) . . . . 138,2 14,20 13,4 $1 \mathrm{~mm} \quad 741,9 \mathrm{~mm} \quad 95,70$

Nach Beendigung der

Diffusion (trocken) * $158,3 \quad 14,1^{\circ} \quad 3,0 \quad 741,9 \quad 111,22$

Die Behandlung der nach der Diffusion zurückbleibenden Mischung mit Alkohol gab folgende Zahlen :

II.

Beobach- Temp. C. Diff, der Barome- Corr. Vol. tetes Vol. Quecksiller- ter bei $0^{\circ} \mathrm{C} .11$.

Angewandles Gas hölıeı 1w Druck

(feucht) . . . 115,1 14,00 $57,2 \mathrm{~mm} \quad \mathbf{7 4 5 , 8 \mathrm { mm } \quad \mathbf { 7 4 , 0 9 }}$ Nach Ausorplion durch

Alkohol . . . . $\quad 56,4 \quad 14,0^{\circ} \quad 81,1 \quad 745,8 \quad 34,32$

Das Gasgemenge enthält demnach in 100 Theilen :

\begin{tabular}{lr} 
Brennbares Gas & 53,68 \\
Almosphärische Luft & $\mathbf{4 6 , 3 2}$ \\
\hline $\mathbf{1 0 0 , 0 0}$
\end{tabular}

Fafst man dieses Resultal mil den an cler Diffusionsrölıre abgelesenen Zahlen (vid. I.) zusammen, so ergiebl sich das Volumen der wälırend der Diffusion eingedrungenen Luft und das des zu gleisher Zeit ausgetretenen Gases, wie folgt :

Eingedrungene Luft $\mathbf{5 1 , 5 2}$

Ausgelrelenes Gas 36,00 .

Die Verbremung der gleichen Mischumg im grofsen Eudiometer lieferte folgende Zalilen :

\section{III.}

Beobach- Temp. C. Diff. der Barome- Corr. Vol. Quecksilber-
höhen ter bol. $\begin{aligned} & \text { bei } 0^{\circ} \mathrm{C} . u . \\ & 1 \text { m Druck }\end{aligned}$

Angewandles Gas höhen

(feucht) . . . $134,6 \quad 14,2^{\circ} \quad 583,2 \mathrm{~mm} \quad 741,3 \mathrm{~mm} \quad 18,68$ 
Beobach- Temp.C. Diff. der Barome- Corr. Vol. tetes Vol. Quecksilber- ter bei $0^{\circ} \mathrm{C}$. u.

Nach Zulassung von höhen $1 \mathrm{~m}$ Druck

Iuft (feucht) $\quad$. $483,7 \quad 14,1^{\circ} \quad 217,9 \mathrm{~mm} \quad 741,6 \mathrm{~mm} 235,35$

Nach Zulassung von

Saucrstoff (feucht) . $\quad 578,5 \quad 14,1^{\circ} \quad 131,2 \quad 741,6 \quad 329,17$

Nach der Verbrennung

(feucht) . . . . $543,8 \quad 13,7^{\circ} \quad 162,2 \quad 742,0 \quad 294,16$

Nach Absorplion der

$\begin{array}{llllll}\mathrm{CO}_{2} \text { (trockell) . - 495,5 } & 13,6^{\circ} & 207,4 & \mathbf{7 4 4 , 8} & \mathbf{2 5 3 , 6 4}\end{array}$

Aus obigen Daten wird berechnet, dafs 18,68 Vol. der Mischung, welche nach (II.) 10,03 Vol. brennbares Gas enthalten, mit 65,50 Vol. Sauersloff zu 40,52 Vol. Kohlensäure verbrennen. Dem entspricht folgendes Verhältnifs :

Vol. des brembaren Gases Verzehrter Sauerstof Erzeugte Kohlensäure $1 \quad: \quad 6,53 \quad: \quad 4,04$.

Das Gas hat demnach in seinem Condensalionsverhällnifs und dem relativen Kohlenstoff - und Wasserstoffgehalt keine Verïnlerung erlitlen, und ist daher für ein einfaches Gas zu halten, wenn man nicht annehmen will, dafs darin zwei oder mehrere Gase gerade in solchen Verhältnissen gemengt enthalten sinl, die ilhren Diffusionscoefficienten entsprechen, ein Fall, der hier unmöglich eingetreten seyn kann. Die obige Methode möchte überhaupt fast in allen Fällen, wo es sich darum handell, zu wissen, ob man es mit einem einfachen Gase oder mit einem Gemenge zu lluun hal, mit Erfolg anwenilbar seyn.

Aus jenen Daten läfst sich das specifische Gewicht des Aelhylgases nach dem bekannlen Graham'schen Geselze ableilen : Jafs die Geschwindigkeit, womit Gase diffundiren, in gcradem Verhältnifs zu deren Volumen steht und sich ungekehrt verhält, wie die Quadratwurzeln ihrer Dichligkeit. Ist daher das Volumen der Luft bekannt, welche in den Diffusionsapparat eingedrungen ist, ferner das ausgetrelene Gasvolumen, 
und bezeichnen wir ersteres mil a, letzteres mit $b$, und das unbekannte specifische Gewicht desselben mit $x$, so erhält man folgende Gleichung :

Hieraus ergiebt sich :

$$
a: b=1: \frac{1}{\sqrt{x}} \text {. }
$$

$$
x=\frac{a^{2}}{b^{2}}
$$

Setzt man die obigen Werthe fïr $a$ und $b$ in diese Gleichung, so erhält man :

$$
\mathrm{x}=\frac{51,52^{2}}{36^{2}}=2,0481 .
$$

Letztere Zahl slimmt mit dem aus der Wägung [2,0462 (S. 178)] und der Berechnung (S.189) sich ergebenden specifischen Gewicht so nahe ïberein, als man nur erwarten kann, wenn man erwägl, wie schwierig es ist, das Gas während der Diffusion unter constantem Almosphärendruck zu erhalten, und dals die Harlnäckigkeit, womil der Gyps Wasser zurückhäl, es fast unmöglich macht, das Gas vor der Ablesung seines Volumens volikommen zu trocknen. Es ist kaum zu bezweifeln, dals die Bestimunungen der specifischen Gewichle von Gasen mit Hülfe eines langen Diffusionseudiometers, welches mit einer zweckmäfsigen Vorrichtung zur Regulirung des Druckes versehen ist, und bei Anwendung einer vollkommen trockmen porösen Substanz anstalt des Gypses sich mit gröfster Schärfe ausführen lassen werden.

Fassen wir die obigen Resultale zusammen, so ergiebt sich daraus die unzweifelhafte Thalsache, dals das Radical Aethyl einen Bestandtheil der Zerselzungsproducte des Jodäthyls ausmacht, in welche letzteres durch Einwirkung von Zink in erhöhter Temperatur zerfällt, und dafs es daraus im Zustande vollkommener Reinheit erhalten werden kann.

Das Aethyl ist ein farbloses, schwach ätherarlig riechendes*) und mit stark leuchtender, weilser Flamme verbrennendes

*) Dieser Geruch ist anfangs sehr slark und rührt wahrscheinlich von 
Gas von 2,00394 specifischem Gewicht. Es enthält in Uebereinstimmung mil den obigen Bestimmungen seines specilischen Gewichts und den eudiometrischen Verbrennungen 2 Volumina Kohlendampf und 5 Vol. Wasserstoff zu 1 Volumen condensirl:

2 Vol. Kohlendampf 1,65844

\begin{tabular}{llll}
5 &, & Wasserstoff & 0,34550 \\
\hline 1, & Aethylgas & 2,00394
\end{tabular}

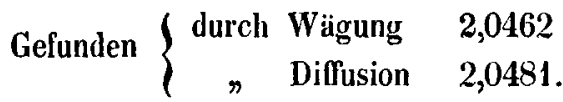

Bei einer 'Temperalur von $-18^{\circ} \mathrm{C}$. wird es noch nicht condensirt, wenn man es mit dem ihm beigemengten Melhyl durch eine, mit einer Kältemischung von jener 'Temperalur umgebene, gewundene Glasröhre langsam streichen läfst; aber bei Anwendung eines 0 erstedt'schen hydrostatischen Condensationsapparates (dessen Benutzung Herr Professor Gerling mir freundlichst gestattete) läist es sich leicht bei $+3^{0} \mathrm{C}$. schon unter einem Drucke von 21 Almosphären in eine farblose, durchsichlige, leicht bewegliche Flïssigkeit verwandeln, welche nach Aufhebung des Drucks ihren früheren gasförmigen Zustand augenblicklich wieder annimmt. Sein Kochpunkt mag daher bei gewühnlichem Druck der Luft etwa bei $-23^{\circ} \mathrm{C}$. liegen. Das Aethylgas ist im Wasser unlöslich, in Alkohol leicht löslich. Ueber Quecksilber mit einer geringen Menge frisch ausgekochten Alkohols zusammengebracht und geschüttelt, verschwand es rasch und vollständig (bis auf ein kleines Luftbläschen, welches kaum 1' Procent vom Ganzen betrug). 1 Vol. absoluter Alkohol alssorbirt bei $14,2^{\circ} \mathrm{C}$. und $744,8 \mathrm{~mm}$ Druck 18,13 Volumina des-

einer in geringer Menge beigemengten fremdarligen Substanz her; nach längerem Steben über Wasser und nachheriger Behandlung des Gases mit rauchender Schwefelsäure verschwindet er beinahe vollständig. Reines Aethyl ist daher gleich den Methyl wahrscheinlich geruchlos. 
selben. Einige Tropfen Wasser dieser Auflösung hinzugefügt, bewirken im ersten Augenblick eine milchige Trübung derselben, gleich darauf fängt das Gas an, daraus zu entweichen und nach wenigen Secunden hat es fast ganz sein anfängliches Volumen wieder eingenommen. Rauchende Schwefelsäure ist ohne Einwirkung auf Aethyl, auch wird es von concentrirter Salpetersäure und Chromsäure nicht merklich verändert, noch verbindet es sich nit Jod oder Schwefel, selbst wenn man diese Körper in dem Gase erhitzt. Der Schwefel bewirkt hierbei eine andere Veränderung des Aethyls, indem sich Schwefelwasserstoff in Menge bildet und Kohle absetzt, sobald dic Temperatur die der Rothgliihhitze erreicht hat. Mlit einem halben Volumen Sauerstoff vermischt und über Plalinschwamm geleitet, bleibt es bei gewölnlicher 'Temperatur unverändert; erst bei Anwendung einer schwachen Hitze fängt der Schwamm an zu glühen; dabei wird unter gleichzeitigem Absalz einer geringen Menge Kohle Wasser gebildet und ein Gas, wahrscheinlich Grubengas, tritt auf, welches in Alkohol unlöslich ist, mit schwach leuchtender Flamme brennt und dahei viel Kohlensäure erzeugt. Gleich deın Methyl wird das Aethylgas von Antimonsuperchlorid nicht absorbirt, auch wenn man es damit dem directen Sonnenlichte ausselzt. Chlor wirkt im Dunkeln nicht darauf ein. Wird aber eine trockene Mischung gleicher Volumina der beiden Gase dem zerstreuten Licht ausgesetzt, so findet augenblicklich eine Vereinigung Statt, zugleich tritt dabei unter Bildung einer farblosen Flüssigkeit eine merkliche Volumveränderung ein. Brom übt ebenfalls eine Einwirkung auf das Aethyl aus, wenn beide dem directen Sonnenlichte ausgesetzt und gelinde erhitzt werden. Ich habe die Untersuchung der hierbei auftretenden Producte für jetzt nicht weiler ausgedehnt, behalte mir aber noch eine ausführlichere Beschreibung dieser Zerselzungserscheinungen vor.

In der Hoffnung, die Bildung des Elayls und Methyls bei der Darstellung des Aethyls verhindern zu können, prüfte ich, 
welchen Einflufs zunächst die Gegenwart von Wasser, dann auch die von Alkohol und Aether bei der Zersetzung des Jodäthyls durch Zink ausüben möchten. Obschon die Erwartung, der Zersetzung des Aethyls hierdurch vorzubeugen, nicht in Erfüllung gegangen ist, so dürften doch die Resultate, welche ich im Folgenden millheilen werde, in anderer Beziehung nicht ohne Interessc seyn.

\section{Einwirlkung des Zinks auf Jodäthyl und Wasser.}

Gleiche Theile Wasser und Jodälliyl mit Zink in cine zuvor evacuirte Glasröhre auf dic oben beschriebene Weise hermelisch cingeschlossen und darauf in einem Oclbade erhilzt, erleiden bei einer noch niederern Temperatur, als Zink und Jodälhyl für sich erfordern, eine rasch fortschreitende Zerselzung. Nach Verlauf von 2 Stunden sclien die Einwirkung beendet zu seyn, der flüssige Inhalt der Röhre wurde dick und erstarrte wälrend des Erkaltens zu einer weilsen amorphen Masse.

Beim nachherigen Oeffnen der Spitze unter Schwefelkalium enthaltendem Wasser strömte eine grofse Menge Gas mit hedeutender Heftigkeit aus. Dasselbe wurde in dem früher beschriebenen Apparate mit den nülhigen Vorsichtsmaafsregeln aufgefangen. Durch Behandlung des in der Röhre bleibenden weilsen Rückstandes mit Wasser liefs sich daraus nicht mehr, wie früher, ein Gas abscheiden.

Die Untersuchung des zuvor gesammelten Gases im kleinen Eudiometer gab folgende Zahlen :

I.

Beobach- Temp.C. Diff. der Barome- Corr. Vol. tetes Vol. Quecksilber- ter bei $0^{\circ} \mathrm{C}$.u.

Angewandtes Gas höhen 1 m Druck

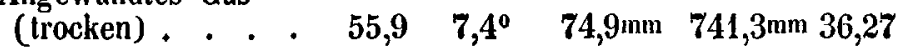

Nach Absorption durch

$\begin{array}{llllll}\text { Schwefels. (trocken) } & 55,9 & \mathbf{7 , 7} & \mathbf{7 3 , 3} & \mathbf{7 3 9 , 8} & 36,24\end{array}$ 
Das Gas wurde so vollständig vom Alkohol absorbirt, dafs der bleibende äufserst geringe Rückstand nicht mehr bestimmt werden konnte.

Die Verbrennung des Gases im grofsen Eudiometer lieferte nachstehende Resultate :

II.

Beobach- Temp, C. Diff. der Barome- Corr. Vol. tetes Vol. Quecksilber- ter bei $0^{\circ} \mathrm{C} . \mathrm{u}$. höhen 1m Druck

Angewandtes Gas

(feucht). . . . $\quad 100,5 \quad 8,0^{\circ} \quad 621,3 \mathrm{~mm} \quad 739,6 \mathrm{~mm} \quad 10,77$

Nach Zulassung von

Luft (feucht) . . $514,1 \quad 8,0^{\circ} \quad 187,9 \quad 739,0 \quad 271,25$

Nach Zulassung von

$\begin{array}{llllll}\text { Sauerstoff (feucht) } & 540,6 & 8,0^{\circ} & 162,7 & 738,5 & 298,21\end{array}$

Nach der Verbrennung

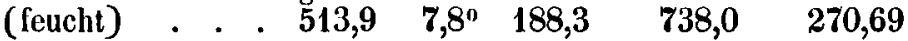

Nach Absorption der

Kohlens. (trocken) $\quad 488,0 \quad 7,6^{\circ} \quad 212,0 \quad 738,1 \quad 249,78$

Nach Zulassung von

Wasserst. (trocken) $\mathbf{7 1 7 , 9} \quad \mathbf{7 , 7 ^ { \circ }} \quad \mathbf{1 0 , 2} \quad \mathbf{7 3 7 , 9} \quad \mathbf{5 0 8 , 0 8}$

Nach der Verbrennung

(feucht) - . . . $612,0 \quad 8,0^{\circ} \quad 100,1 \quad 737,8 \quad 374,40$

Angewandies Gas

$\begin{array}{lllllll}\text { (feucht) . . . } & \text {. } & 155,0 & 8,2^{\circ} & 558,6 & 737,8 & 25,75\end{array}$

Nach Zulassung von

Luft (feucht) - . $\quad 521,2 \quad 8,0^{\circ} \quad 182,1 \quad 737,3 \quad 277,08$

Nach Zulassung von

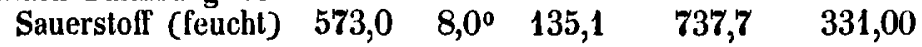

Nach der Verbrennung

(feucht) . . . . $509,1 \quad 7,8^{\circ} \quad 193,1 \quad 738,0 \quad 265,79$

Nach Absorption der

Kohlens. (trocken) $\quad 449,6 \quad 7,9^{\circ} \quad 246,2 \quad 738,8 \quad 215,24$

Nach Zulassung von

Wasserst. (trocken) $665,1 \quad 8,1^{0} \quad 52,0 \quad 737,9 \quad 443,04$

Nach der Verbrennung

(feucht) . . . $\quad 629,2 \quad 8,5^{\circ} \quad 82,7 \quad 736,9 \quad 394,12$ 
Aus der Bestimmung I. ergiebt sich, dafs das untersuchte Gas keine Spur von Elayl beigemengt enthält, und ferner aus

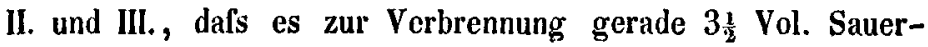
stoff bedarf, um damit 2 Vol. Kohlensäure zu erzeugen :

\begin{tabular}{|c|c|c|c|c|}
\hline \multirow[t]{2}{*}{ Analyse } & II & $\begin{array}{c}\text { Gasvolum } \\
10,77\end{array}$ & $\begin{array}{c}\text { Verbrannter Sauersto } \\
37,66\end{array}$ & $\begin{array}{c}\text { Erzeugte Kohle } \\
20,91\end{array}$ \\
\hline & $=$ & 1 & 3,50 & 1,94 \\
\hline & III & 25,75 & 90,01 & 50,55 \\
\hline & $=$ & 1 & 3,50 & 1,97 . \\
\hline
\end{tabular}

Genau dieselben Resultate hat die Analyse des Methyls *) ergeben, mit dem es überhaupt in seinen Eigenschaften völlig übereinslimmt. Es ist farblos, fast ganz unlöslich in Wasser, löslich in Alkohol, welcher bei $8,8^{\circ} \mathrm{C}$. und $665,5 \mathrm{~mm}$ Druck 1,22 Volumina davon aufnimmt; es besitzt anfangs einen schwachen ätherartigen Geruch, der nach Behandlung mit Alkohol und Schwefelsäure völlig verschwindet, und wird selbst bei einer Temperatur von $-18^{\circ} \mathrm{C}$. nicht flüssig. Chlor isl im Dunkeln ohne Wirkung darauf, wird aber die Mischung dem zerstreuten 'Tageslichte ausgesetzt, so sieht man die Farbe des Chlors rasch verschwinden, ein Beweis, dafs eine Vereinigung slattgefunden hat. Es verbindet sich weder mit Jod noch mit Schwefel, selbst wenn diese Körper in dem Gase erhilzt werden.

Es unterliegt daher keinem Zweifel, dafs das durch Einwirkung von Zink auf Jodäthyl bei Gegenwart von Wasser entstehende Gas reines Methyl und identisch ist mit demjenigen, welches durch Zersetzung des Cyanäthyls vermitlelst Kalium irit] und durch Elektrolyse der Essigsäure ***) erhallen ist.

Ein Versuch, das Methyl durch Compression in den tropfbarflüssigen Aggregatzusland üherzuführen, hat nicht das ge-

*) Diese Annalen Bd. LXV, S. 275 und Bd. LXIX, S. 290.

**) Ebendaselbst Bd. LXV, S. 269.

***) Ebendas. Bd. LXIX, S. 279. 
wünschte Resultat gegeben, wenigstens war, bei einem Druck von 20 Almosphären (dem höchsten, welchen das mir zu Gebole stehende Instrument anzuwenden gestattete) und gleichzeitiger Tempcratur von $+3^{\circ} \mathrm{C}$, keinc Spur von ciner Condensation sichlbar.

Die weifse Salzmasse, wclche in der Zersclzungsröhre zuriickblieb, besafs cinen starken Geruch nach Aether; sic entlicll indefs kcinen organischen Körper in chemischer Verbindung, sondern bestand aus basischem Jodzink.

Die Bildung des Melhyls durch Einwirkung von Zink auf Jodiathyl und Wasser läfst sich durch folgende Gleichung veranschaulichen :

$$
\mathrm{C}_{4} \mathrm{H}_{5} \mathrm{~J}+\mathrm{HO}+2 \mathrm{Zn}=2\left(\mathrm{C}_{2} \mathrm{H}_{3}\right)+\mathrm{ZnJ}, \mathrm{ZnO} \text {. }
$$

Um darzulhun, dals dic Zerselzung des Jodäthyls in keinem anderen Sinne erfolgt, und namentlich dafs dabei das Aethyl, wie man vielleicht annehmen könnte, sich nicht in Melhyl und Elayl spaltet, welches letzlere damn in irgend einer chemischei Verbindung im Rückstande enthalten seyn müfstc, habe ich folgenden Versuch angestellt :

Eine gewogene Menge Jodäthyl (2,268 Grm.) wurde wie vorhin in einer hermelisch verschlossenen Röhre mil Zink und Wasser möglichst vollständig zersetzt, die Rölıre nach dem Erkalten geölfnet und der Rückstand, nachdem alles Gas ausgetreten war, rasch mil kaltem Kupferoxyd gemengt und darauf in einer Verbrennungsröhre wie bei der organischen Analyse crhitzt. Die Gewichlszunahme des Kaliapparates betrug 0,132 Grm., einem Gehalt von 0,036 Grm. Kohlenstoff entsprechend. Die gefundene Kohlensloffmenge belrägt aber nur $\frac{1}{5}$ von derjenigen, welche in dem Rückstande vorhanden seyn müfste, wenn einc Zerlegung des Jodäthyls in Jodzink, Methyl und Elayl slattgefunden hätle. Bei dieser Voraussetzung hälte näinlich der Rïickstand von 2,268 Grın. Jodälhyl, welcher dann dic enlsprechende Menge Elayl in chemischer Verbindung enthalten 
würde, statt der gefundenen 0,132 Grm. Kohlensäurc, 0,641 Grm. (= 0,175 Grm. C.) geben müssen. Ahgesehen hiervon, liefert schon die Anwesenheit der grofsen Menge von Zinkoxyjodid im Rückstande einen hinlänglichen Beweis, dals eine Wasserzerselzung staltgefunden hat und dafs daher 1 Atom Jodälhyl, nach Abscheidung des Jods, durch Aufnalime von Wasserstoff geradeauf in 2 Atome Methyl verwandelt ist *).

Diese Thatsache erhält eine fernere Bestätigung durch die Zersetzung, welche Jodäthyl und Wasser erleiden, wenn man sie für sich in einer hermelisch verschlossenen Röhre derselben Temperatur aussetzt, welche zur Bildung des Methyls erforderlich ist. Man findet sie beim Oeffnen der erkalteten Röhre in Aether und eine concentrirte Lösung von Jodwasserstoffsäure verwandelt. Diese Aetherbildung giebt zugleich Aufschlufs über die Gegenwart desselben in der bei obiger Darstellung des Methyls zurückbleibenden Salzmasse, wie über die bei der Verbrennung derselben mit Kupferoxyd erhaltene geringe Menge von Kohlensäure.

Die beobachtete Zersetzung des Jodälhyls durch Zink bei Gegenwart von Wasser gewährt eine sehr einfache Methode, sich vollkommen reines Melhylgas in grofser Menge zu ver-schaffen, indem schon ein einziges Zersetzungsrohr von den oben angegebenen Dimensionen davon mehr als $300 \mathrm{Cbc}$. liefert.

Um sich vor den Folgen der beim Erhilzen der Röhren möglicher Weise eintretenden Explosionen zu schützen, ist es ralhsam, den Apparat mit einem hölzernen Behälter zu umgeben, welcher hinten offen und vorn mit einem doppelten Glasfenster versehen ist, wodurch sich der fortschreitende Gang der Operation beobachten läfst. Die Quantität des in den Zersetzungsröhren von den obigen Dimensioncn zu zerlegenden Jodäthyls

*) Ueber die Einwirkung des Zinks auf Jodäthyl bei Gegenwart von Wasser und Alkohol finden sich weitere Angaben in der nachstehenden Notiz ,über eine neue Reihe organischer Verbindungen" etc. 
darf nicht 3,5 Grm. und die Temperatur des Oelbades nicht $180^{\circ}$ uibersteigen. Uebrigens ist mir im ganzen Verlaufe dieser Untersuchung keine einzige Glastöhre durch eine Explosion zertrümmert, obschon sie milunter einen Druck von 80 bis 100 Atmosphären zu ertragen halten.

\section{Einwoirkung des Zinks auf Jodäthyl und Alkohol.}

Wenn unan dic lelzt beschriebenen Versuche dahin abändert, dafs man statt des Wassers absoluten Alkohol mit Zink und Jodäthyl in der verschlossenen Röhre crhitzt, so erhält man nach dem Oeffnen derselben fast genau dieselbe Menge Gas, wie vorhin. Auch liefs sich aus dem Rückslande, in welchem sich die Gegenwart von Aether durch den Geruch leicht zu erkenmen gab, beim Uebergiefsen mit Wasser kein Gas mehr gewinnen.

Die eudiometrische Analyse führte zu folgenden Resultaten:

I.

Beobach- Temp.C. Diff. der Barome- Corr. Vol. tetes Vol. Quecksilber- ter bei $0^{\circ} \mathrm{C} . \mathrm{u}$.

Augewandtes Gas

(feucht) . . . . 122,7 $8,8^{\circ} \quad 6,1 \mathrm{~mm} \quad 732,3 \mathrm{~mm} \quad 86,32$

Nach Behandlung mit

$\begin{array}{llllll}\text { Schwefels. (trocken) } & 120,9 & 8,2^{\circ} & 8,2 & 740,0 & 85,89\end{array}$

Nach Herausnahme eines

Theils zur Verbrennung (lrocken) * . $\quad 84,3 \quad 9,0^{\circ} \quad 43,8 \quad 732,6 \quad 56,21$

Nach Absorplion durch

Alkoho

$3,1 \quad 8,6^{\circ} \quad 31,9 \quad 732,1 \quad 2,06$

II.

Angewandtes Gas

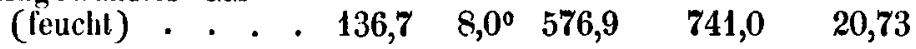

Nach Zulassung von

atm. Luft (feucht) . $\quad 504,0 \quad 8,3^{\circ} \quad 192,6 \quad 741,3 \quad 264,37$ 
Beobach- Temp.C. Diff. der Barome- Corr. Vol. tetes Vol. Quecksilber- ter bei $0^{\circ}$ C.u.

Nach Zulassung von höhen $1 \mathrm{~m}$ Druck

Sauerstoff (feucht) $\quad 574,6 \quad 8,4^{\circ} \quad 128,7 \mathrm{~mm} \quad 741,1 \mathrm{~m} \cdot \mathrm{n} \quad 336,81$ Nacb der Verbrennung

(feucht) . . . . 526,3 $8,2^{\circ} \quad 172,3 \quad 740,9 \quad 286,39$

Nach Absorplion der

Kohlensäure (trocken) $482,3 \quad 7,9^{\circ} \quad 213,2 \quad 738,2 \quad 246,08$

Nach Zulassung von

Wassersloff (trocken) $702,0 \quad 8,0^{\circ} \quad 23,7 \quad \mathbf{7 3 7 , 7} \quad 486,95$

Nach der Verbrennung

(feucht) . . . . $566,1 \quad 8,1^{\circ} \quad 140,9 \quad 737,3 \quad 323,44$

Aus dem Versuch I. ergiebt sich die Abwesenheit von Elayl und ein Gchalt von 3,66 Procent Stickstoff. Nach Versuch II. ist das Verhältnils des brennbaren Gases zum verbrannten Sauerstoff und der erzeugten Kohlensäure wic folgt :

Angewandtes Gas Verbrannter Sauterstoff Erzeugte Kohlensäure

$\begin{array}{rrrrr}19,97 & 70,76 & & 40,31 \\ 1 & : & 3,54 & : & 2,02 .\end{array}$

Das Gas hat daher dieselbe Zusammenselzung und Dichtigkeit, wie das durch Einwirkung von Zink auf Jodäthyl und Wasser erhaltene Methyl, mit dem es auch in seinen physikalischen Eigenschaften übereinstimmt. Die Gegenwart von basischem Jodzink und Aether im Rückstande machen es unzweifelhaft, dafs 1 Aeq. Jodälhyl sich mit 1 Aeq. Alkohol und 2 Aeq. Zink in 2 Aeq. Methyl, 1 Aeq. Aether und 1 Aeq. Zinkoxyjodid zerlegt :

$$
\begin{array}{lll}
\mathrm{C}_{4} & \mathrm{H}_{5} & \mathrm{~J} \\
\mathrm{C}_{4} & \mathrm{H}_{5} & \mathrm{O}, \mathrm{HO} \\
& 2 & \mathrm{Zn}
\end{array} \mid \begin{array}{ll}
2 & \left.\begin{array}{ll}
\left(\mathrm{C}_{2}\right. & \mathrm{H}_{3}
\end{array}\right) \\
\mathrm{C}_{4} & \mathrm{H}_{5} \mathrm{O}
\end{array}
$$

Einwirkung des Zinks auf Jodäthyl und Aether.

Gleiche Volumina Jodäthyl und Aelher mit Zink in einer hermetisch verschlossenen Röhre bei ohngefähr $150^{\circ} \mathrm{C}$. so lange erhitzt, bis die Zersetzung beendet schien, gaben nach dem 
Erkallen eine dickllüssige, ölige, nicht crstarrende Masse. Bein Abbrechen der Spitze entwichen nur wenige Cubikzoll Gas; dagegen trat beim Uebergiefsen des Rückstandes mit Wasser eine desto leblıaftere starke Gasentwickelung cin. Beide Gase wurden in demselben Gefäfs aufgefangen und der cudiometrischen Analyse unterworfen.

I.

Beobach- Temp.C. Diff. der Barome- Corr. Vol. tetes Vol. Quecksilber- ter bei $0^{\circ}$ C. u.

Angewandtes Gas höhen $\quad 1^{m}$ Druck

(trocken) . . . + 185,2 12,90 19,7m $744,2 \mathrm{~mm} \quad 128,12$

Nach Absorption durch

$\mathrm{SO}_{3}$ (Irocken) · . $175,1 \quad 13,0^{\circ} \quad 4,3 \quad 741,4 \quad 123,20$

Nach Herausnahme eines

Theils zur Verbren-

nung (trocken) . . 105,7 14,30 65,8 $745,9 \quad 68,31$

Nach Absorption durch

Alkohol . . . . $\quad 7,4 \quad 14,1^{\circ} \quad 71,2 \quad 746,4 \quad 4,58$

II.

Angewandtes Gas

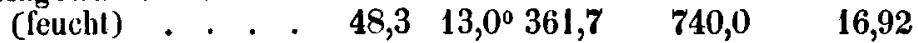

Nach Zulassung von

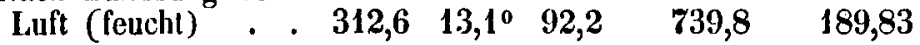

Nach Zulassung von

Sauerstoff (feucht) . $\quad \begin{array}{lllll}355,5 & 12,7^{\circ} & 48,4 & 738,8 & 230,82\end{array}$

Nach der Verbrennung

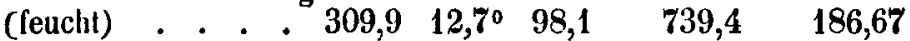

Nach Absorption der

Kohlensäure (trocken) $260,5 \quad 12,9^{\circ} \quad 144,6 \quad 738,3 \quad 147,68$

Nach Zulassung von

Wasserstoff (trocken) $388,1 \quad 13,0^{\circ} \quad 14,8 \quad 737,9 \quad 267,87$

Nach Zulassung von:

$\begin{array}{llllll}\text { Sauerstoff (trocken) } & 427,2 & 13,3^{\circ} & 12,8 & 737,3 & 295,12\end{array}$

Nach der Verbrennung

(fencht) . . . . $302,0 \quad 13,4^{0} \quad 102,4 \quad 737,1 \quad 179,40$ 
Der Versuch I. führt zu folgender Zusammensetzung :

Durch $\mathrm{SO}_{3}$ absorbirbares Gas . . . 3,84

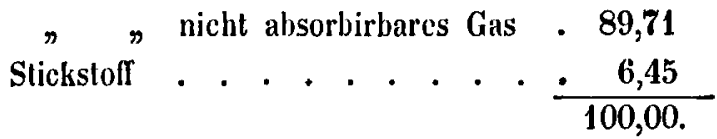

Das Verbällnifs des nach Absorplion durch Schwefelsäure zurückbleibenden Gases zu der für scine Verbrennung erforderliclıen Sauerstoffmenge und der erzeugten Kohlensäure ist uach Versuch II. wie folgt :

Vol. des brennbaren Gases Verbrannter Saucrstoff Erzengtc Kohlensäture

$\begin{array}{rrrrr}15,83 & & 67,31 & & 38,99 \\ = & : & 4,25 & : & 2,46 .\end{array}$

Aus jenem Zahlenverhältnifs geht hervor, dals das von Schwefelsäure nicht absorbirbare Gas cin Gemenge ist und dafs der eine Beslandtheil ein höheres Alomgewicht als das Methyl besilzl. Die Auflöslichkeit dieses Gemenges in Alkohol, wclcher nur cine geringe Menge Stickstoff ungelöst zurückläfst, schliefst übrigens die Gegenwart von Wasserstoff und Grubengas aus. Wenn man die Gegenwart des Elayls in Betrachl zieht, wclches durch Schwefelsäure zuvor absorbirt wurde und zugleich das Verhalten des Jodälhyls gegen Zink sowohl für sich, wie bei Gegenwart von Wasser und Alkohol berücksichtigt, so kann man nicht zweifelhaft seyn, dafs das fragliche brennbare Gasgemenge aus Aethyl und Methyl besteht. Durch Anwendung der oben entwickelten Formeln findet man, dars 15,83 Vol. desselben 4,57 Vol. Aelhyl und 11,26 Vol. Methyl enthalten. Läfst man den zufälligen Gehalt an Slickstoft unberiicksichtigt, so führen beide Analysen zu folgender procentischcr Zusammensetzung der gesammlen Mischung :

$$
\begin{array}{lr}
\text { Aethyl } & 27,68 \\
\text { Melhyl } & 68,22 \\
\text { Elayl } & 4,10 \\
\hline & 100,00 .
\end{array}
$$


Ich behalte mir vor, die Zersetzung des Jodäthyls durch Zink bei Gegenwart von Aether genauer zu studiren und namentlich zu untersuchen, in welcher Verbindung diese Gase in dem Aether enthalten sind, welche sich bein Vermischen mil Wasser daraus entwickeln.

Ich will zum Schlusse mit ein paar Worten noch einiger Versuche erwähnen über das Verhalten des Jodälhyls zu anderen Melallen; es mag genügen, die beobachteten Erscheinungen kurz zu beschreiben.

Die Metalle: Eisen, Blei, Kupfer und Quecksilber bei einer von 150 bis $200^{\circ} \mathrm{C}$. variirenden Temperalur mit Jodäthyl erhitzt, zersetzen kaum cine Spur davon. Dagegen wird es von Arsenik bei circa $160^{\circ} \mathrm{C}$. rasch zerlegt, indem sich eine blutrothe Flüssigkeit erzeugt, welche beim Erkalten in prachtvollen Krystallen, wahrscheinlich Arsenjodid (As $\mathrm{J}_{3}$ ), anschiefst. Beim Oeffnen der capillaren Spitze der Röhre erwies sich dieselbe luftleer; auch entwickelte die krystallinische Masse in Contact mit Wasser, worin sie sehr wenig löslich ist, kein Gas, Das übrig gebliebene Arsenik besafs im auffallend lohen Grade cinen starken, dem des Anlimons sehr ähnlichen Metallglanz. Zinn bewirkt dic Zcrlegung des Jodäthyls beinahe bei derselben Temperalur, wie Arsenik, und las fliissige Jodid verwandelt sich dabei allıälig in cin gelbliches, öliges Liquidum, welches beim Erkalten krystallinisch erslart. Eine Gasentwickelung fand weder beim Oeffnen der Röhre, noch bei nachleriger Behandlung des Rückstandes mil Wasser Statt, welches kaum eine Spur davon löste. Auch von Kalium wird das Jodälhyl bei einer Temperatur von ungefähr $130^{\circ} \mathrm{C}$. mit Leichtigkeit zersetzt, die Zersetzungsproducte sind denen, welche man durck Zink erhält, entsprechend; da jedoch die Kaliumkugeln stets mit einer Schicht Kalihyc. at bedeckt sind und es kaum möglich ist, sie ohne diese in die Zersetzungsröhre einzuführen, so enthalten die Gase stets mehr oder weniger freien Wassersloff. 
Ich enthalte mich, an die vorstebenden Beobachtuagen, so sehr sie dazu Veranlassung geben, allgemeine Betrachtungen über die wahrscheinliche Constilution der Radicale der Reihe, welcher das Aelhyl und Melhyl angehören, anzuknüpfen, bis ich meine Versuche auf einige andere Glieder derselben und auf die elektronegaliven Radicale, das Formyl, Acetyl etc. weiter ausgedehnt habe.

Noliz über eine neue Reihe organischer Körper, welche Metalle, Phosphor u. s. w. enllalten; von Dr. E. Frankland.

Seil Beendigung der vorhergehenden Untersuchung über die Einwirkung von Zink auf Jodäthyl, habe ich das Verhalten von Zink gegen die entsprechende Methylverbindung studirt; die Resultate, welche ich in einer späleren Abhandlung mittheilen werde, sind den andern nahezu analog : es entwickelt sich Methylgas und in der Zersetzungsröhre bleibt ein weilser, lirystallinischer Rückstand. Das eigentluümliche Verhalten dieses Rückstandes gegen Wasser, durch welches er unter einer glänzenden Flammenerscheinung, mit Entwickelung von reinem Sumpfgas zerselzt wird, veranlafste mich, diese Zerselzung genauer zu untersuchen. Unterwirft man den Rückstand in einem mil trocknem Wasserstoffgas gefülten Apparate der trocknen Destillalion, so geht eine farblose, durchsichtige Flüssigkeit von ausnehmend durchdringendem und äufserst widrigem Geruch, in die Vorlage über; diese Flüssigkeit entzündet sich von selbst, wenn sie mit Luft oder Sauersloffgas in Berührung kommt, und verbrennt mit einer glänzenden, grünlich-blauen Flamme unter Bildung einer dichten Wolke von Zinkoxyd; der 